\title{
A Framework for Optimizing the Cost and Performance of Next-Generation IP Routers
}

\author{
Henry C. B. Chan, Hussein M. Alnuweiri, Member, IEEE, and Victor C. M. Leung, Senior Member, IEEE
}

\begin{abstract}
The explosive growth of Internet users, the increased user demand for bandwidth, and the declining cost of technology have all resulted in the emergence of new classes of high-speed distributed IP-router architectures with packet-forwarding rates on the order of gigabits, or even terabits, per second. This paper develops an analytical framework for modeling and analyzing the impact of technological factors on the cost-performance tradeoffs in distributed-router architectures. The main tradeoff in a distributed router results naturally from moving the main packet forwarding and processing power from a centralized forwarding engine to an ensemble of smaller forwarding engines, either dedicated to or shared among the line cards. Processing packets in these smaller engines can be much cheaper (by as much two to three orders of magnitude) than in a centralized forwarding engine. Therefore, the main goal of our modeling framework is to determine an optimal allocation of processing power to the forwarding engines (in a distributed router) to minimize overall router cost while achieving a given level of packet-forwarding performance. Two types of router models are analyzed using the proposed framework: a distributed-router architecture and parallel-router architecture.
\end{abstract}

Index Terms - Cost optimization, distributed router, IP networks, IP routers, parallel router, performance optimization, router architecture.

\section{INTRODUCTION}

$\mathbf{T}$ HE last three years have witnessed a rising interest in high-performance routers as a very viable alternative to frame- or cell-forwarding switches in campus and backbone networks [1]-[8]. ${ }^{1}$ Driven by the insatiable demand for bandwidth by customers and by the decreasing cost of technology, new generations of switched routers that support service differentiation over gigabit links have started to emerge [2], [4]. ${ }^{2}$ Also, recent innovations in fast-route lookup through table compaction [9], [10], hardware-assisted table lookup [10]-[12], or hashing-based techniques [13] have contributed to overcoming one of the last hurdles toward building gigabit, or even terabit, routers. By distributing route-lookup oper-

Manuscript received July 14, 1998; revised January 30, 1999. This work was supported by a Strategic Project Grant from BC Advanced Systems Institute and PMC-Sierra, Inc.

H. C. B. Chan was with the Department of Electrical and Computer Engineering, University of British Columbia, Vancouver, B.C. V6T 1 Z4 Canada $\mathrm{He}$ is now with the Department of Computing, Hong Kong Polytechnic University, Hung Hom, Kowloon, Hong Kong.

H. M. Alnuweiri and V. C. M. Leung are with the Department of Electrical and Computer Engineering, University of British Columbia, Vancouver, B. C. V6T 1 Z4 Canada.

Publisher Item Identifier S 0733-8716(99)04481-9.

${ }^{1}$ See http://www.pluris.com/tech.htm.

${ }^{2}$ See http://www.packetengines.com/products/pr5200; http://www.zdnet. com/zdtag/snci/reports/packetengines.pdf; and http://www.ascend.com/230. html. ations and through the use of high-capacity, space-division switching, vendors have been able to offer routers capable of forwarding over 40 million packets per second ( $\mathrm{p} / \mathrm{s})$ with delays not exceeding $27 \mathrm{~ms}^{3}{ }^{3}$ Such routers are known as switched routers or routing switches because they normally employ a switching fabric and distributed architecture for forwarding packets across the router ports. These routers are also called layer-3 switches, wire-speed routers, or hardware-based routers. Although such routers are demonstrating impressive performance, the economics of such hardware-based routers have not been well investigated. It is the purpose of this paper to explore this issue in some detail.

Packet forwarding in traditional routers was implemented in software running on one (or a few) general-purpose processor(s). In such routers, all packets arriving on any of the router line card (LC) interfaces go through the central processing pool for route calculation and forwarding to the output links. The centralized processing feature of traditional routers has led such routers to become one of the major bottlenecks in the expanding Internet. As link rates started to increase to the gigabit/second range, routers had to deal with forwarding millions of packets per second from each LC. To meet the increasing demands on routers, a number of enhancements have been proposed and implemented in recent routers. Most of the enhancements involve distributing the functionality of the forwarding engines among multiple smaller forwarding engines that can be implemented in specialized hardware or with recently advanced general-purpose processors. Other enhancements to the basic-router architecture include replacing the shared-bus interconnect by a space-division switching fabric that can handle data rates reaching up to several hundred gigabits/second. Indeed, commercial routers with an aggregate (input-plusoutput) rate of $100 \mathrm{Gbit} / \mathrm{s}$ have been reported recently [4]. ${ }^{4}$

With the emergence of these new classes of switched routers comes the question of cost versus performance, which is an issue of primary importance, especially for the enterprise networks where the goal is to provide connectivity to a large number of end points at the lowest possible cost. This paper develops an analytical framework for modeling and analyzing the impact of technological factors on the costperformance tradeoffs in distributed-router architectures. The main tradeoff in a distributed router results naturally from moving the main packet forwarding and processing power from a centralized forwarding engine to an ensemble of smaller forwarding engines either dedicated to or shared among the

\footnotetext{
${ }^{3}$ See http://www.packetengines.com/products/pr5200 and http://www zdnet.com/zdtag/snci/reports/packetengines.pdf.

${ }^{4}$ See http://www.packetengines.com/products/pr5200.
} 

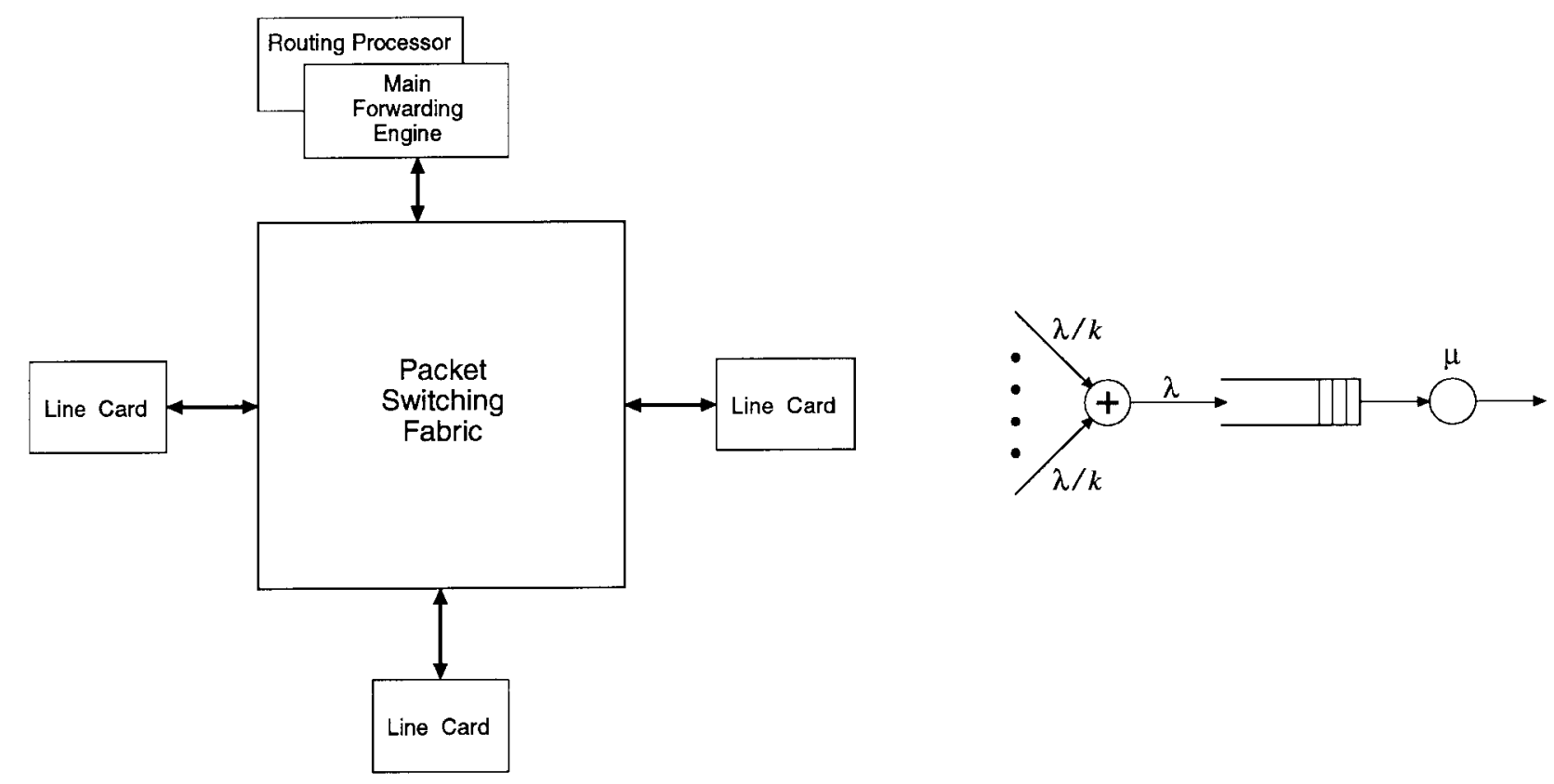

Fig. 1. Centralized router architecture.

LC's. Processing packets in these smaller engines can be much cheaper (by as much 100-1000 times) than in a centralized forwarding engine. Therefore, the main goal of our modeling framework is to determine an optimal allocation (or partition) of processing power of the forwarding engines (in a distributed router) so as to minimize overall router cost while achieving a given level of packet-forwarding performance. In this paper, cost is based almost entirely on the relative processing power of the forwarding engines. This can be justified by observing that it is the innovations in the forwarding engines that drive the current generation of gigabit routers and contribute mostly to their cost. Almost all other system components remain relatively fixed per router port and per unit bandwidth.

In the following sections, we will focus our discussion on two models of high-performance router architectures. These two models embody two major trends in the design of current high-end commercial routers.

\section{Distributed- AND PARAllel- Router ARChitectures}

As mentioned earlier, router architectures have evolved to incorporate distributed and parallel implementations of the packet-forwarding process. The basic structure and operation of such routers is explained in the following sections. In this section, we will begin by providing an overview of the architecture and operation of a basic conventional router.

Traditional router architecture was centered around a network processor and a forwarding engine consisting of a routing processor that performs most of the routing and packetforwarding operations with the help of a master routing table maintained by the network processor. Among other things, the network processor is responsible for periodically updating the master routing table and for implementing a host of routing protocols (e.g., RIP, BGP, OSPF, IGMP, etc. [1]). The organization of a typical traditional router is shown in Fig. 1. It consists of a plurality of LC's, each supporting a number of link-layer interfaces, such as Sonet OC-3, Sonet OC-12, Gigabit Ethernet, Fast Ethernet, etc. Each LC normally includes a simple satellite processor and a small cache of recently used routes. The processor and cache deployed in the LC's utilize the possible locality in packet headers to forward packets directly to the outbound LC's without going through the main forwarding engine. Packets whose header cannot be located in the LC cache are forwarded to the main forwarding engine. In reality, only packet headers need to be exchanged between the LC's and the main forwarding engine; complete packets are transferred among LC's only. As shown in Fig. 1, the various router components are interconnected by a switch fabric, which in earlier routers consisted of a shared (backplane) bus. Packet forwarding through a router starts at the LC's. When an LC receives a packet, it forwards the packet to the forwarding engine for processing. The forwarding engine then looks up the route for that packet, rewrites the packet header (e.g., by updating the MAC address and recalculating the error control checksum), and sends the header back to the originating LC together with the outbound LC address. The input LC can then forward the packet to the outbound LC. The most time-consuming part of this process is the route lookup in the forwarding engine, since this requires searching through a large database of destination prefixes (e.g., 100000 routes) for the maximum-length prefix that matches the destination field of a given packet. Without one of the fast route-lookup techniques mentioned before, the performance of conventional routers was severely limited by this operation. To speed up the route lookup, a cache is normally employed [11]. It basically stores some routes that have been recently looked up to shorten the processing time for certain packets. The performance of a conventional router is highly dependable on the cache hit rate. In general, if all route lookups miss a cache, the performance of a conventional router can be degraded by as much as $50 \%$. 

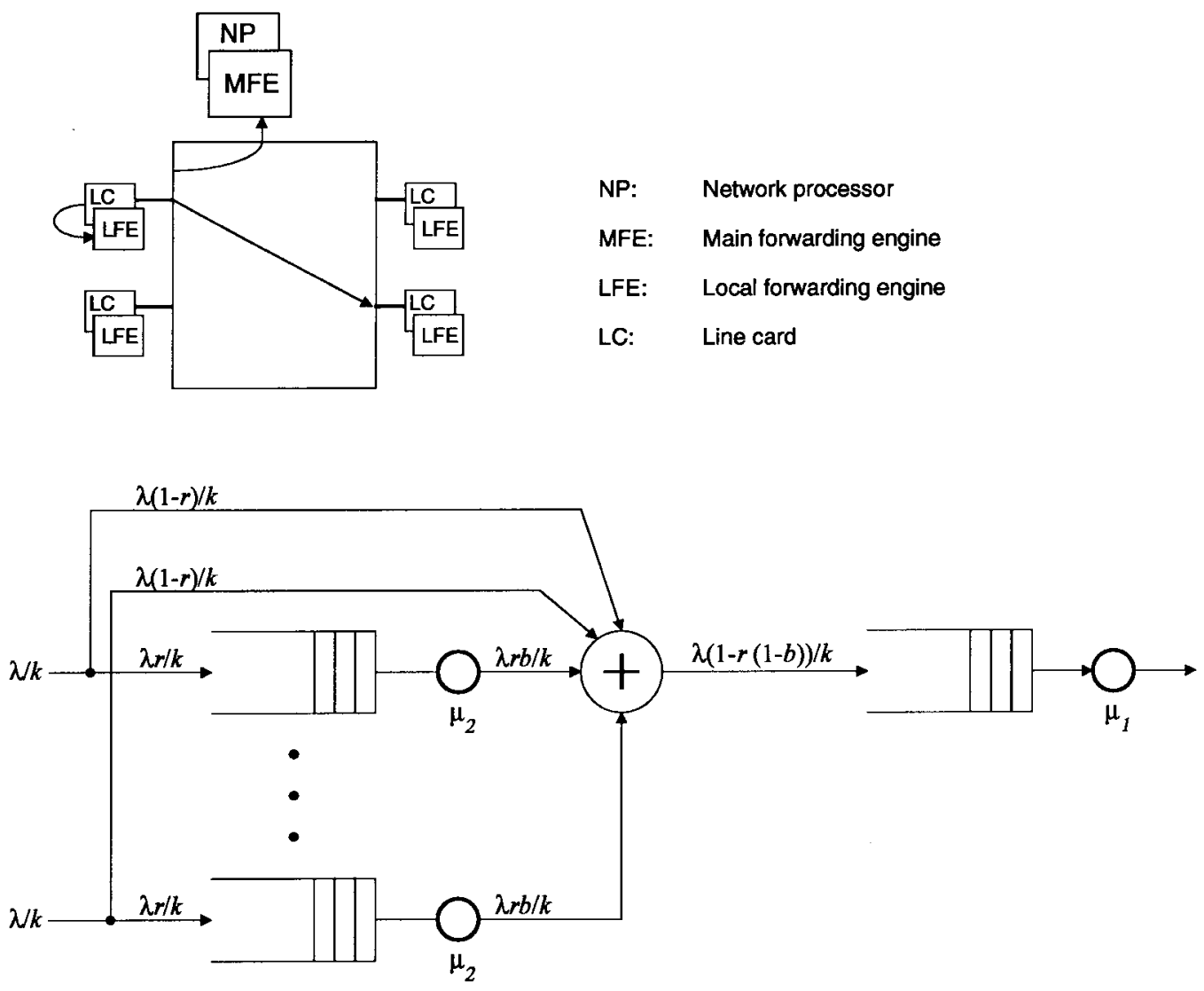

Fig. 2. Distributed-router architecture.

In the following section, we will focus our discussion on two major models of high-performance, distributed-architecture routers. These two models embody two major trends in the design of current high-end commercial routers, as will be argued in Section II-A.

\section{A. Distributed-Router Architecture}

The first router model that we will introduce is the distributed-router architecture that is illustrated in Fig. 2. The main components of this router are LC's, forwarding engines, and a switching fabric. Note that the router is supplied with two types of forwarding engines: a main forwarding engine (MFE) and several local forwarding engines (LFE's), each associated or integrated with an LC. The MFE contains the master routing table and a powerful routing processor, or even a multiprocessor. This router model encompasses such commercial routers as the PowerRail 5200 from Packet Engines, Inc., ${ }^{2}$ Cisco's Catalyst $8510,{ }^{5}$ and the GigaRouter from NetStar (now, Ascend Communications, Inc.). ${ }^{6}$ However, our model is more general in that it allows packet forwarding to be performed by the LFE's or the MFE, based on local route availability, as will be explained in Section II-B.

Fig. 2 also shows a queuing model of the distributedarchitecture router. In this model, each LC has a forwarding engine to process a fraction $r$ of the route lookups. When a

\footnotetext{
${ }^{5}$ See http://www.zdnet.com/zdtag/snci/reports/cisco8510.pdf.
}

${ }^{6}$ See http://www.ascend.com/230.html. packet is received, it will either be processed locally by the LFE or transferred to the MFE with probabilities $r$ and $1-r$, respectively. The routing processor periodically downloads a routing table to each LFE. If a route is found by the MFE or by an LFE, the packet header is rewritten accordingly. If a route cannot be found by an LFE, e.g., due to an outdated route table or a packet that needs special processing, the packet will be transferred to the MFE. It is assumed that a route can always be resolved at the MFE. A major advantage of this architecture is that most packets can be forwarded directly from the incoming LC to the outgoing LC, whereas in the centralized architecture, all packets have to be forwarded twice through the router.

\section{B. Parallel-Router Architecture}

The second router model is the parallel-router architecture, which maintains a bank of forwarding engines (of equal power) separately from the LC's, as illustrated in Fig. 3. The key feature of this model is that it allows the forwarding engines to be shared among the LC's, thus increasing the port density of the routers. Also, decoupling the forwarding engines from the LC's has the advantage of allowing the packetforwarding rate to be increased in a scalable manner until it meets the aggregate capacity of high-speed links, especially in backbone routers. This can be achieved by adjusting the ratio of the number of forwarding engines to that of LC's. A minor disadvantage can be caused by the LC's having to forward the header of each packet they receive to a forwarding engine that 


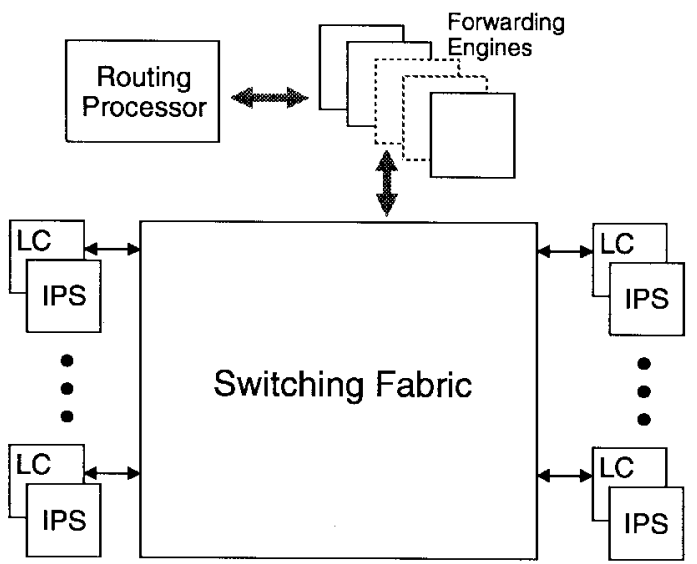

IPS: IP-switching controller

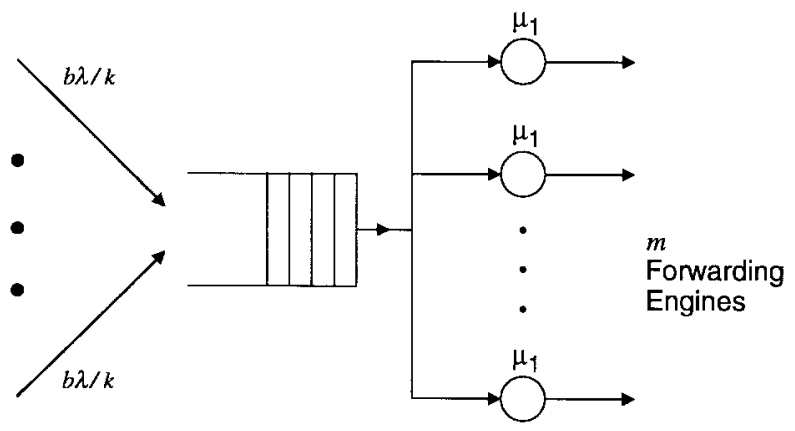

Fig. 3. Parallel-router architecture.

rewrites the new header and sends it back to the appropriate LC for final routing to an outbound LC. This strategy has been adopted in the multigigabit router (MGR) [4] and the Bell Labs experimental router [3].

Fig. 3 illustrates the basic architecture of a parallel architecture, which aims at providing a highly scalable router architecture. A pool of parallel MFE's is located at the routing processor. All MFE's are assumed to have the same copy of routing tables at any time. To minimize the number of packets that traverse the switching fabric within a given time window, a client/server model is employed. Basically, when a packet is received at the LC, a route-lookup request is made to the pool of MFE's. Having received a response, the LC that originated the request modifies the packet header accordingly, then transfers the packet directly to the destination LC. As an option to further reduce the route-lookup load, IP switching [5] can be used at the LC to transport long-lived traffic. IP switching works by caching route-lookup information in the LC's, so that consecutive packets from the same identified flow can be forwarded directly to output LC's without performing repeated route-lookup operations. Note that IP switching can also be applied to the distributed-router architecture described earlier. We emphasize its use for the parallel-router architecture because combining IP switching and parallel-route lookup potentially leads to very high-performance routing switches. IP switching can be implemented in routers at a small additional cost.
In Section III, we develop a cost model for the two router architectures previously described. The cost model will be incorporated in a queuing model, which will then be used to determine an optimal allocation (or partition) of processing power among the various types of forwarding engines within the same router, so as to minimize router cost while achieving a given packet-forwarding rate. The earlier allocation problem will actually be formulated as an optimization problem that will be solved by analytical and numerical techniques in Sections III and IV.

\section{Cost-Optimization Model}

In this section, we present a cost-optimization model for the previously mentioned distributed- and parallel-router architectures. In fact, many high-speed routers are designed based on similar architectures ${ }^{7}$ [4], [5]. The models will be used to asses the impact of certain design parameters on the router cost. For example, currently the cost of retrieving a route from the main forwarding engine is more expensive than processing packet headers in the local forwarding engines by two to three orders of magnitude. However, as interconnect costs and shared-memory access hardware costs are coming down, the difference in cost between local and nonlocal access may diminish. To make the analysis feasible, we need to make a number of simplifying assumptions, such as Poisson arrival rate, exponential distributed lookup time, etc. Although these assumptions have certain limitations in modeling the real situation, our results still provide valuable insight into investigating router-design trends. In particular, our work provides a framework to investigate the cost effectiveness of emerging distributed and parallel routers and to quantify the impact of various system parameters on hardware cost and performance. As far as we know, very little work has been done before in this area.

To simplify the analysis, we ignore transmission and switching delays and assume that route lookup is the main cause of delay while forwarding a packet inside a router. The router is assumed to have $k$ LC's, and packet arrivals at each LC are Poisson with a mean of $\lambda / k \mathrm{p} / \mathrm{s}$ so that the mean aggregate arrival rate at the router is $\lambda \mathrm{p} / \mathrm{s}$. Since all packets require a route lookup, the lookup-request rate in each LC is also $\lambda / k$ requests/s. The route lookup times for MFE and LFE are exponentially distributed with means $1 / \mu_{1}$ and $1 / \mu_{2} \mathrm{~s}$, respectively. In other words, the MFE and LFE can process an average of $\mu_{1}$ and $\mu_{2} \mathrm{p} / \mathrm{s}$, respectively. The Poisson assumption can be relaxed or replaced by more realistic packet-arrival models without affecting the applicability of our techniques. The particular packet-arrival model used will only impact the choice of the parameters $\mu_{1}$ and $\mu_{2}$, which determine the relative processing power of the local and main forwarding engines.

A key parameter in our model is the average packetprocessing rate parameter $\mu$, which provides a simple, yet very effective, measure of the processing power of a forwarding

\footnotetext{
${ }^{7}$ See http://www.pluris.com/tech.htm; http://www.packetengines.com/ products/pr5200; http://www.zdnet.com/zdtag/snci/reports/packetengines.pdf; and http://www.zdnet.com/zdtag/snci/reports/cisco8510.pdf.
} 
engine. Because we assume that the cost of a forwarding engine is a function of its processing power, we can write the cost functions $\operatorname{cost}_{\mathrm{MFE}}=f_{1}\left(\mu_{1}\right)$ for the MFE and $\operatorname{cost}_{\mathrm{LFE}}=f_{2}\left(\mu_{2}\right)$ for the LFE. We also assume that the router cost is dominated by the costs of the forwarding engines. This is justified by the fact that the state of the art processing and memory resources are always allocated to the forwarding engines. Other costs such as LC cost, switch-fabric cost, and routing-processor cost are fixed for a given router configuration. In this paper, we employ a simple linear function for expressing the cost of a forwarding engine in terms of its processing power. Thus, we assume that cost $_{\mathrm{MFE}}=c_{1} \mu_{1}, \operatorname{cost}_{\mathrm{LFE}}=c_{2} \mu_{2}$, and the total router cost is cost $=c_{1} \mu_{1}+k c_{2} \mu_{2}$. The parameters $c_{1}$ and $c_{2}$ are the normalized costs for processing packets in the respective forwarding engine. Rather than using absolute measures of cost, our results will be expressed in terms of the relativecost parameter $a=c_{1} / c_{2}$, which is affected mainly by technological factors. This parameter will be used in the following sections to derive cost functions that are based almost entirely on the relative processing power of the forwarding engines. This can be justified by observing that it is the innovations in the forwarding engines that drive the current generation of gigabit routers and contribute mostly to their cost. Almost all other system components remain relatively fixed per router port and per unit bandwidth. However, in a more elaborate model, such additional normalized cost factors can be incorporated in the parameters $c_{1}$ and $c_{2}$.

Due to technological limitations, the processing power of a forwarding engine cannot grow to infinity. The processingpower limits for MFE and LFE are set to be $\mu_{\max 1}$ and $\mu_{\max 2}$ $\mathrm{p} / \mathrm{s}$, respectively. To facilitate the queuing analysis, we assume that all buffers have unlimited capacity so that no packet is lost. Another constraint is concerned with processing delay, where we assume that the average processing delay of a route-lookup operation $(T)$ must not exceed a given maximum $\left(T_{\max }\right)$.

\section{A. Distributed-Router Architecture}

The queuing model for the distributed-router architecture is represented by $\mathrm{M} / \mathrm{M} / 1$ queues as shown in Fig. 2. By using the average delay for an $\mathrm{M} / \mathrm{M} / 1$ queue [15], the average route-lookup delay can be expressed as

$$
\begin{aligned}
T= & \frac{r(1-b)}{\mu_{2}-((r \lambda) / k)} \\
& +r b\left(\frac{1}{\mu_{2}-((r \lambda) / k)}+\frac{1}{\mu_{1}-\lambda(1-r)-r b \lambda}\right) \\
& +\frac{1-r}{\mu_{1}-\lambda(1-r)-r b \lambda}
\end{aligned}
$$

where $b$ is the fraction of route lookups that cannot be resolved locally by the LFE. In other words, they need to be resolved by the MFE. The first, second, and third terms of (1) correspond to the case where a route lookup is to be processed only by the LFE, both LFE and MFE, and only the MFE, respectively. Note that the equation is valid because the output process of an $\mathrm{M} / \mathrm{M} / 1$ queue is also a Poisson process. Equation (1) can be simplified to

$$
T=\frac{r}{\mu_{2}-((r \lambda) / k)}+\frac{1+r b-r}{\mu_{1}-\lambda(1-r)-r b \lambda} .
$$

The objective of the optimization problem is to minimize the total cost i.e.,

$$
\text { minimize } c_{1} \mu_{1}+k c_{2} \mu_{2}
$$

which is equivalent to finding the minimum value of the following objective cost function (OCF) or finding the optimal OCF value (OCFV)

$$
\text { minimize } a \mu_{1}+k \mu_{2}
$$

where $a=\left(c_{1} / c_{2}\right)$. The optimization problem is subject to the following delay and maximum processing-power constraints

$$
\begin{aligned}
& \lambda(1-r)+r b \lambda<\mu_{1}<\mu_{\max 1} \\
& \frac{r \lambda}{k}<\mu_{2}<\mu_{\max 2} \\
& T=\frac{r}{\mu_{2}-((r \lambda) / k)}+\frac{1+r b-r}{\mu_{1}-\lambda(1-r)-r b \lambda} \leq T_{\max } \text {. }
\end{aligned}
$$

As $\mu_{1}$ and $\mu_{2}$ have continuous values, an equality sign can be taken in (7) for finding the optimal solution. This means the constraint actually becomes

$$
T=\frac{r}{\mu_{2}-((r \lambda) / k)}+\frac{1+r b-r}{\mu_{1}-\lambda(1-r)-r b \lambda}=T_{\max } .
$$

Specifically, the objective of the optimization model is to find an optimal set of $\left\{\mu_{1}^{*}, \mu_{2}^{*}, r^{*}\right\}$ values for minimizing the OCFV while meeting the above constraints. Note that the above model is in fact a generalized model for the centralized and distributed architecture. If the optimal solution is $\left\{\mu_{1}^{*}, \mu_{2}^{*}=0, r^{*}=0\right\}$, it means that a centralized architecture is to be used. In the case $r^{*}=1$, it will be more cost effective to pass the route-lookup request to LFE's first. If $r^{*}=1$ and $b=0$, i.e., the LFE's are sufficiently powerful to resolve all route requests, then the architecture will be completely distributed.

If $\mu_{\max 1}$ and $\mu_{\max 2}$ are unbounded while $r$ remains fixed, it is possible to obtain closed-form solutions for the optimal values $\mu_{1}^{*}$ and $\mu_{2}^{*}$. This is done by deriving an expression for $\mu_{1}$ in terms of $\mu_{2}$ using (8) and then using the result to rewrite (4) in terms of $\mu_{2}$ only. By differentiating the OCF with respect to $\mu_{2}, \mu_{2}^{*}$ and $\mu_{1}^{*}$ can be computed as follows.

For $r=0$, the centralized-router architecture case, we have

$$
\mu_{1}^{*}=\frac{1}{T_{\max }}+\lambda .
$$

For $r=1$, the distributed-router architecture case (i.e., all route lookups are first processed through the LFE's), we have

$$
\begin{aligned}
& \mu_{1}^{*}=\frac{b}{T_{\max }\left(1-\frac{1}{1 \sqrt{(a b) / k}}\right)}+b \lambda \\
& \mu_{2}^{*}=\frac{1+\sqrt{(a b) / k}}{T_{\max }}+\frac{\lambda}{k} .
\end{aligned}
$$

In general, it is possible to derive an optimal solution using numerical methods. We have written a Matlab program to 


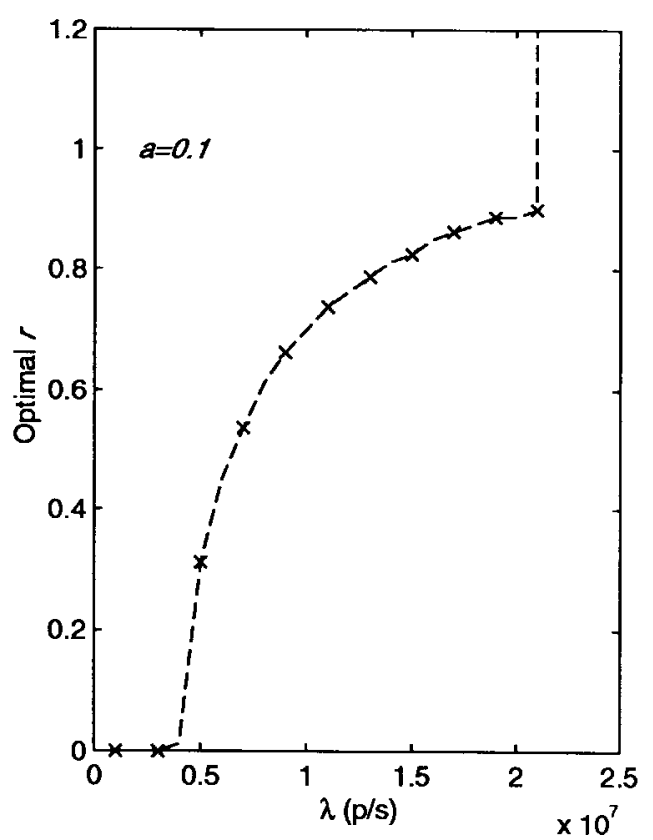

(a)

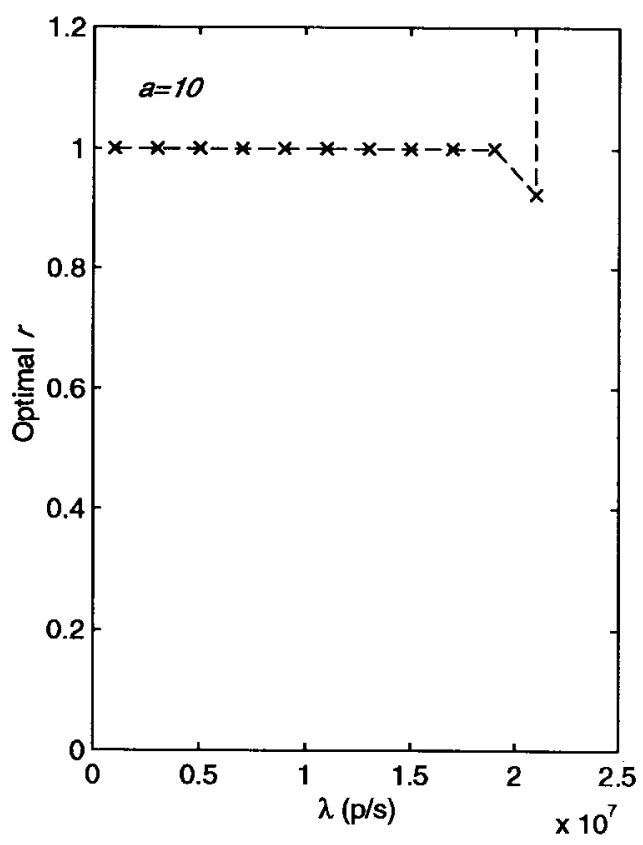

(c)

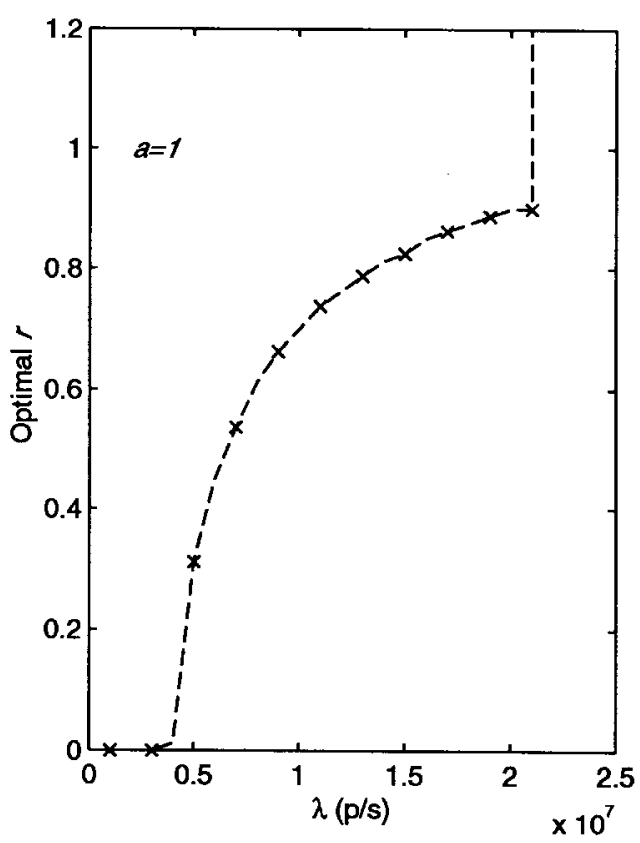

(b)

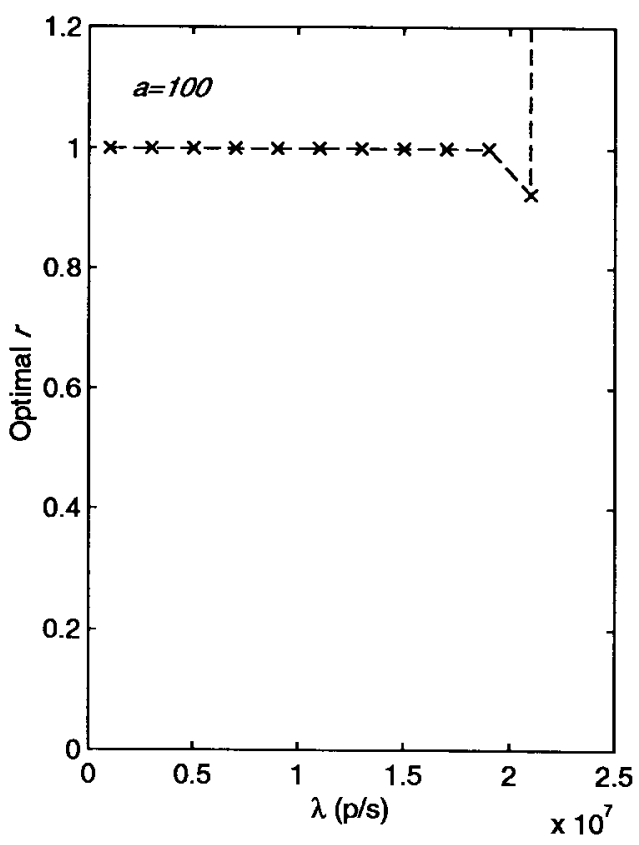

(d)

Fig. 4. Optimal $r$ when $a$ is varied. (a) $a=0.1$. (b) $a=1$. (c) $a=10$. (d) $a=100$.

solve the problem. The method is to choose a feasible value for $\mu_{1}$ and $r$ and then to find $\mu_{2}$ by using (8). Based on the result, the OCFV is evaluated. If $\mu_{2}$ cannot satisfy the constraint, the OCFV is set to $10^{20}$, representing infinity. By varying $\mu_{1}$ and $r$ in very small steps within the feasible ranges, a set of solutions $\left\{\mu_{1}, \mu_{2}\right.$, and OCFV $\}$ are obtained. The optimal solution can then be estimated from the set of solutions (i.e., the one with the lowest OCFV).

\section{B. Parallel-Router Architecture}

The queuing model for the parallel-router architecture is given in Fig. 3, which basically consists of an $\mathrm{M} / \mathrm{M} / \mathrm{m}$ queue.
Again we assume that all buffers have unlimited capacity to enable a simpler queueing analysis. As an option, we consider that IP switching can be used at the LC's to further reduce the route lookups. Effectively, this changes the arrival rate from $\lambda$ to $b \lambda(0<b \leq 1)$ because when a switched path is established, the corresponding packets can be switched through the router fabric with minimal processing time. Note that when $b=1$, it means that IP switching is not activated.

The average route lookup time for is given by [15]

$$
T=\frac{1}{\mu_{1}}+\frac{\rho P_{Q}}{\lambda b(1-\rho)}
$$




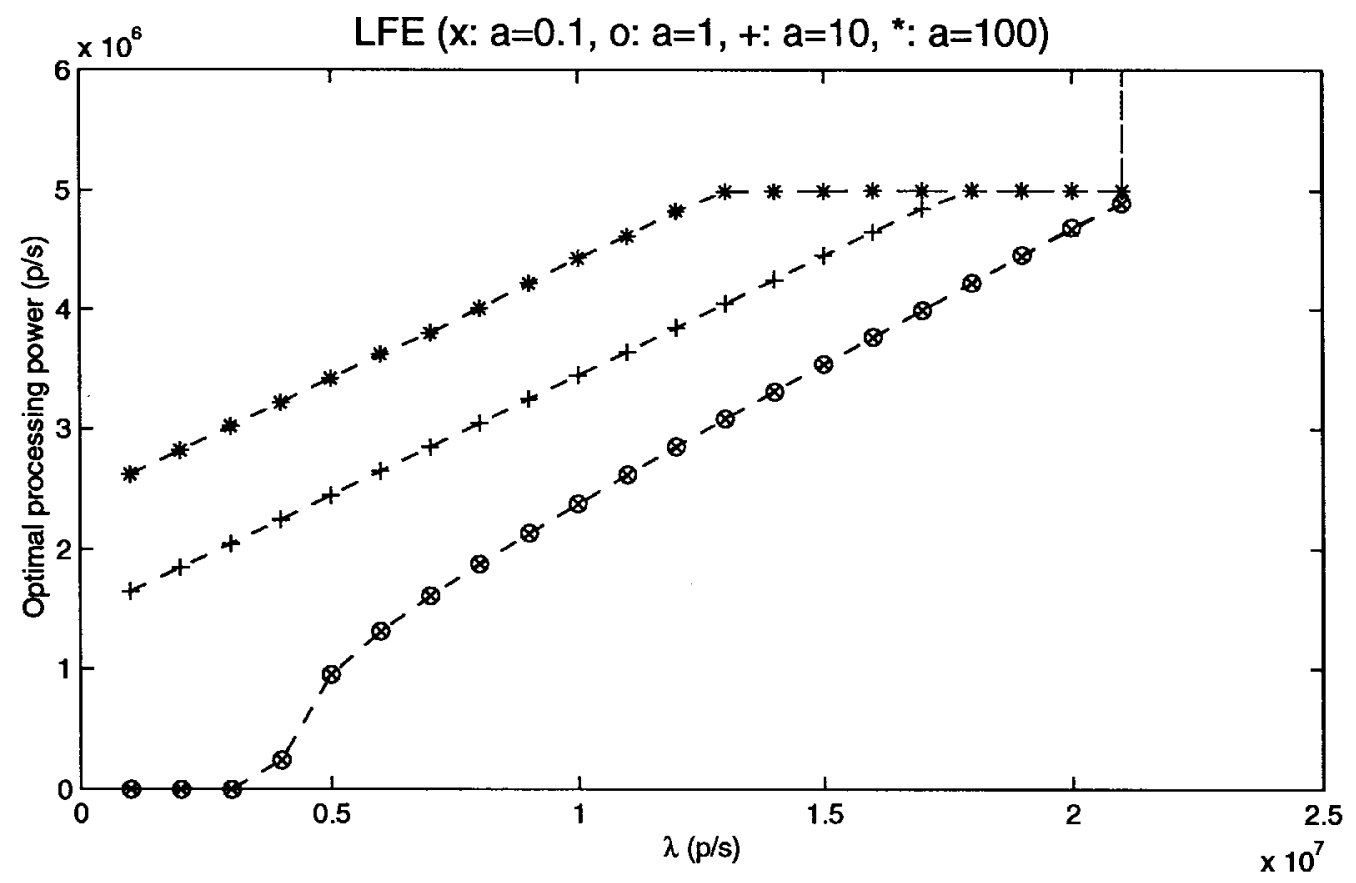

(a)

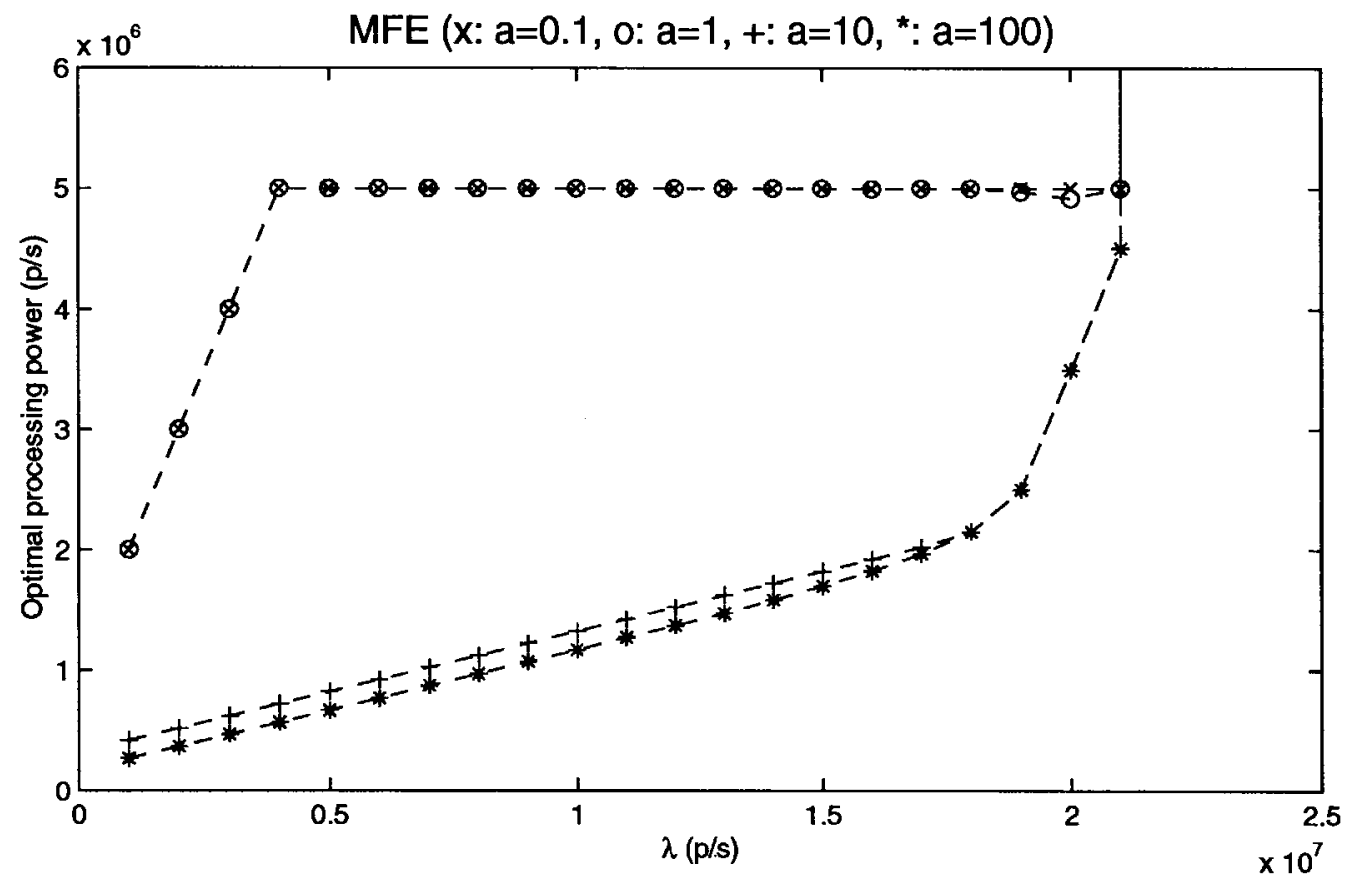

(b)

Fig. 5. Optimal processing power when $a$ is varied. (a) LFE. (b) MFE.

where

$$
\begin{aligned}
\rho & =\frac{\lambda b}{m \mu_{1}} \\
P_{0} & =\frac{1}{\sum_{j=0}^{m-1} \frac{(m \rho)^{j}}{j}+\frac{(m \rho)^{m}}{m !(1-\rho)}} \\
P_{Q} & =\frac{P_{0}(m \rho)^{m}}{m !(1-\rho)}
\end{aligned}
$$

The objective of the cost optimization model is to minimize the following $\mathrm{OCF}$

$$
\text { minimize } m a \mu_{1}
$$

subject to the following processing power and processing delay constraints

$$
\begin{aligned}
\mu_{1} & <\mu_{\max 1}, \\
T & =\frac{1}{\mu_{1}}+\frac{\rho P_{Q}}{\lambda b(1-\rho)}=T_{\max }
\end{aligned}
$$

and $m$ is an integer. The optimal solution $\left\{\mu_{1}^{*}, m^{*}\right\}$ is obtained using Matlab based on the technique just described. 


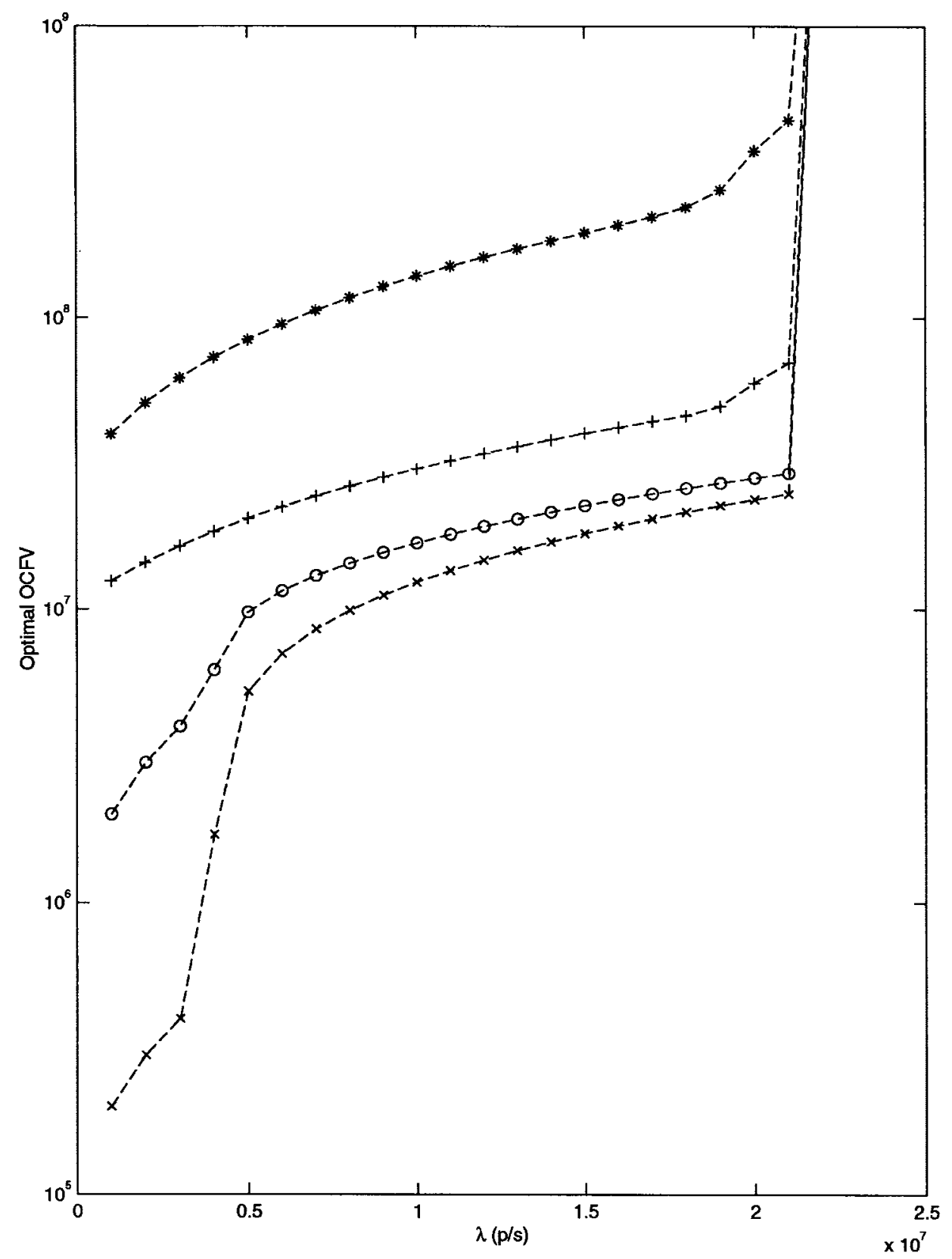

Fig. 6. Optimal OCFV when $a$ is varied (X: $a=0.1$; o : $a=1 ;+: a=10 ; *: a=100$ ).

\section{NUMERICAL RESUlTS AND Discussions}

In this section, we present some numerical results for the router cost-optimization problem. As an example, we define the baseline parameters as follows: the number of LC's in router $k=5$, the processing power of MFE is $\mu_{\max 1}=5$ million $\mathrm{p} / \mathrm{s}$, and the processing power of LFE is $\mu_{\max 2}=5$ million $\mathrm{p} / \mathrm{s}$. Also, we assume that $T_{\max }=10^{-6} \mathrm{~s}, \lambda=5 \times 10^{6}$ $\mathrm{p} / \mathrm{s}$, and $a=10$. With today's technology, the processing power we have presented for MFE and LFE should be feasible [5].

\section{A. Distributed-Router Architecture}

Before presenting the numerical results, we first analyze the closed-form solutions expressed by (10) and (11), which correspond to the case when both the MFE and LFE's can have unlimited processing power, and all route requests are processed in the LFE's first, i.e., $r=1$. Interestingly, it will become evident from the numerical solutions that $r=1$ is in fact the optimal solution in most cases. Therefore, the closedform solutions are useful for predicting the general form of the optimal solution. As (10) and (11) indicate, the functions $\mu_{1}^{*}$ and $\mu_{2}^{*}$ vary linearly with $\lambda$ if the other parameters remain constant. The gradients of these linear functions are $\left(\partial \mu_{1}^{*} / \partial \lambda\right)=b$ and $\left(\partial \mu_{2}^{*} / \partial \lambda\right)=(1 / k)$, respectively. As will be shown later, the general optimal solutions also follow this form even when the MFE and LFE's have limited processing power. In the following section, we present several sensitivityanalysis results that elucidate the impact of varying key parameters on router cost and performance. 


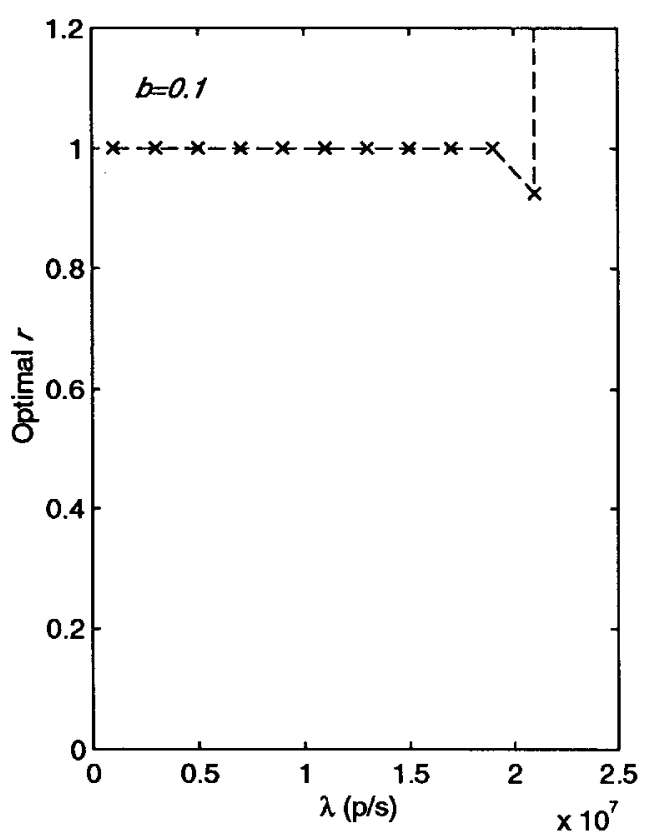

(a)

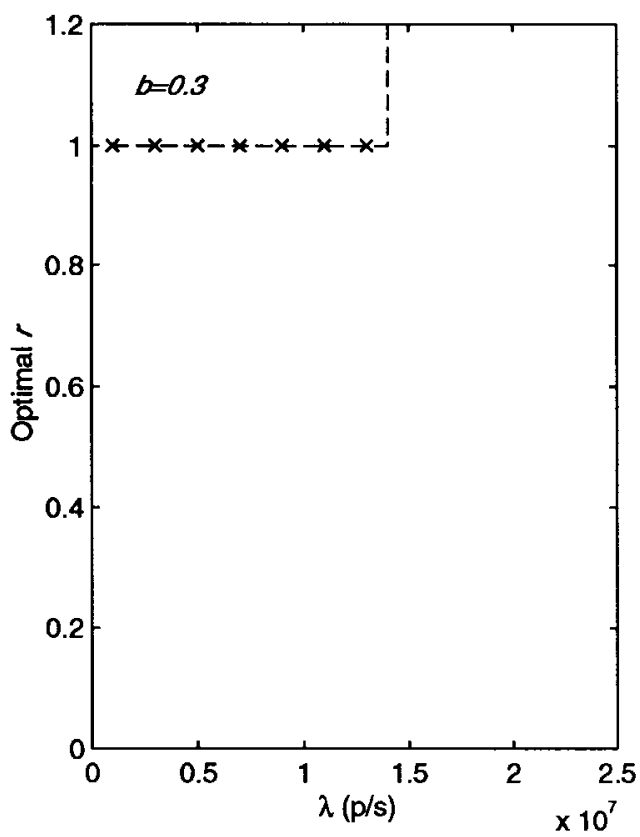

(c)

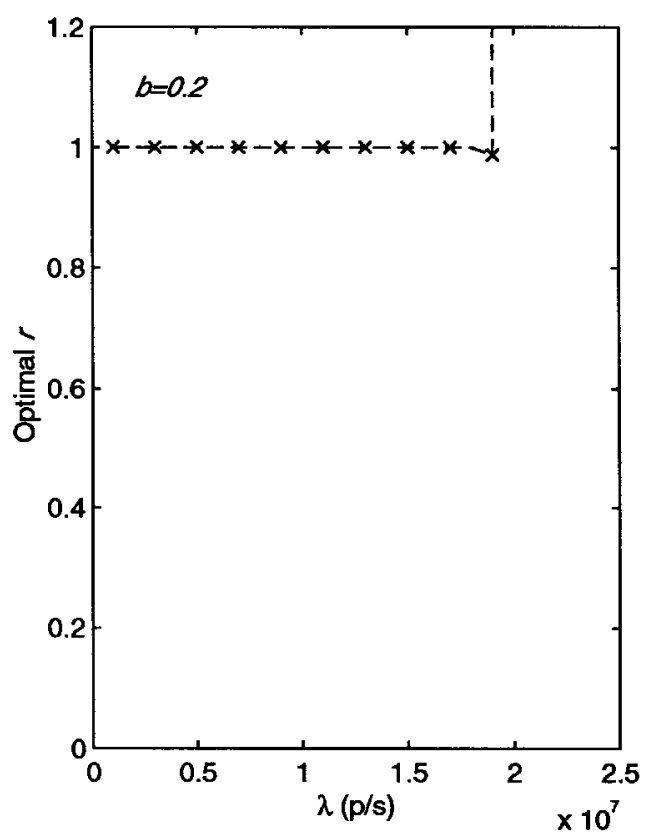

(b)

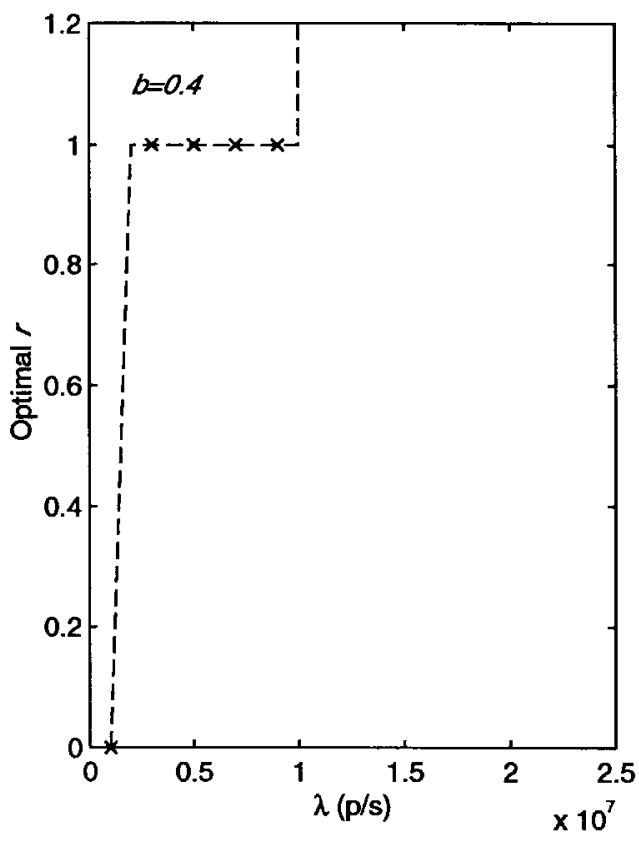

(d)

Fig. 7. Optimal $r$ when $b$ is varied $(a=10)$. (a) $b=0.1$. (b) $b=0.2$. (c) $b=0.3$. (d) $b=0.4$.

1) Impact of the Relative Cost Parametera: Fig. 4 shows plots for the optimal $r$ (the fraction of packets handled by an LFE) when the parameter $a$ is varied (recall that $a$ is the ratio of the cost of an MFE to the cost of an LFE). Two types of results can be deduced from the plots in Fig. 4.

When $a \leq 1$, which is the case when the cost of an MFE is lower than (or close to) that of an LFE, it is more cost effective to use a centralized architecture (hence, $r=0$ ), provided that the MFE can handle the route-lookup load, i.e., $\lambda \leq 5 \times 10^{6}$. If $\lambda$ is increased beyond the capability of a single MFE, the LFE's must be added to share part of the route lookups.

When $a>1$, which is the case when the cost of an MFE is greater than that of an LFE, the optimal solution mandates that all route lookups must be processed locally by the LFE's first (i.e., $r=1$ ), before they are sent to the MFE. The only exception to this is when $\lambda$ is close to the maximum processing limit of the router, in which case it is more cost effective to have the MFE share about $10 \%$ of the route lookups. In our model, the parameter $r$ is set to infinity if there is no feasible solution. Therefore, the plots in Fig. 4 indicate that the router can handle a maximum load of about 20 million p/s.

Fig. 5 shows the optimal processing power of an LFE and an MFE as functions of the target-packet processing $\lambda$ rate and for different values of the parameter $a$. Note that the optimal processing power for an LFE is zero or very small when $a \leq 1$, and the packet rate $\lambda$ is within the processing 


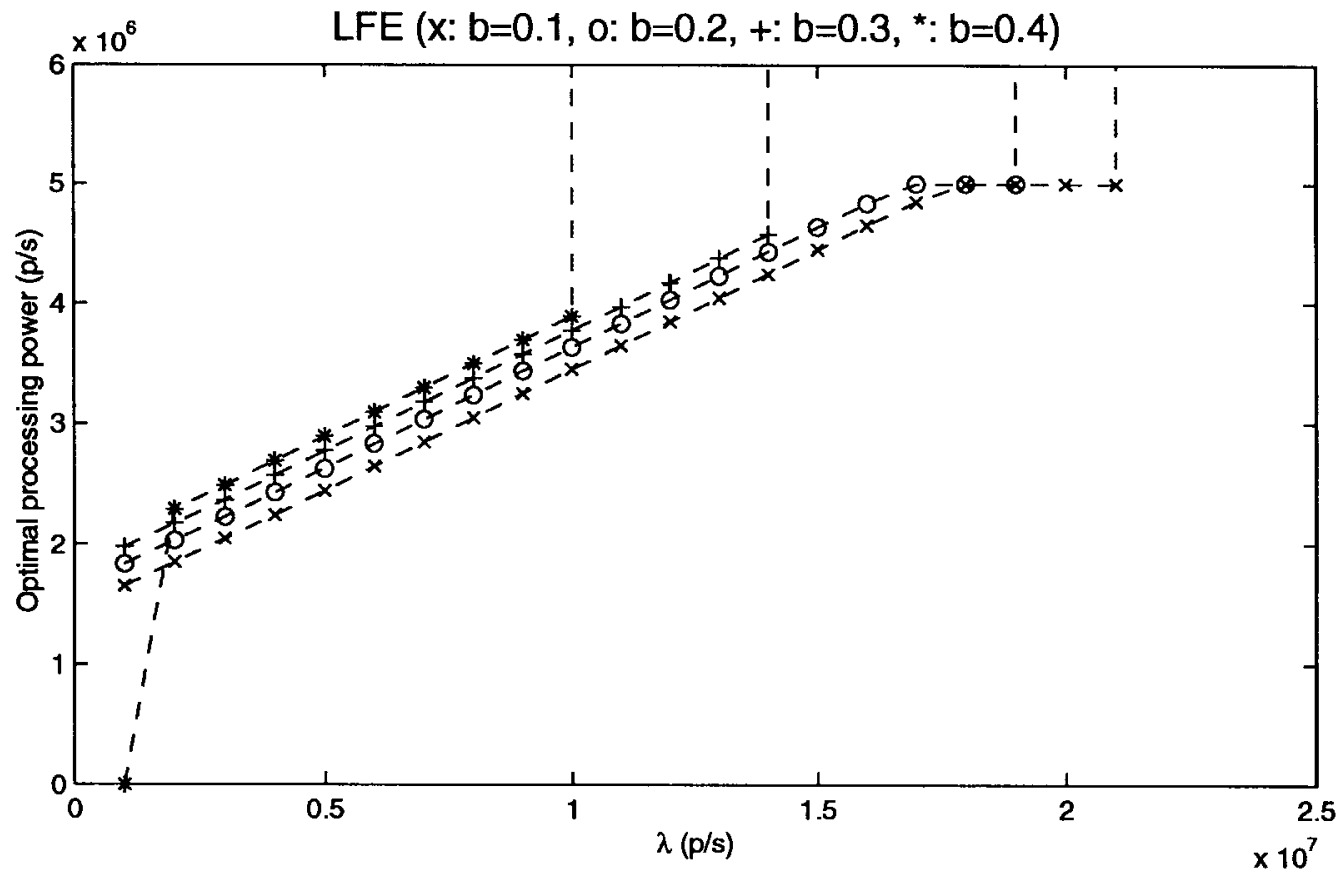

(a)

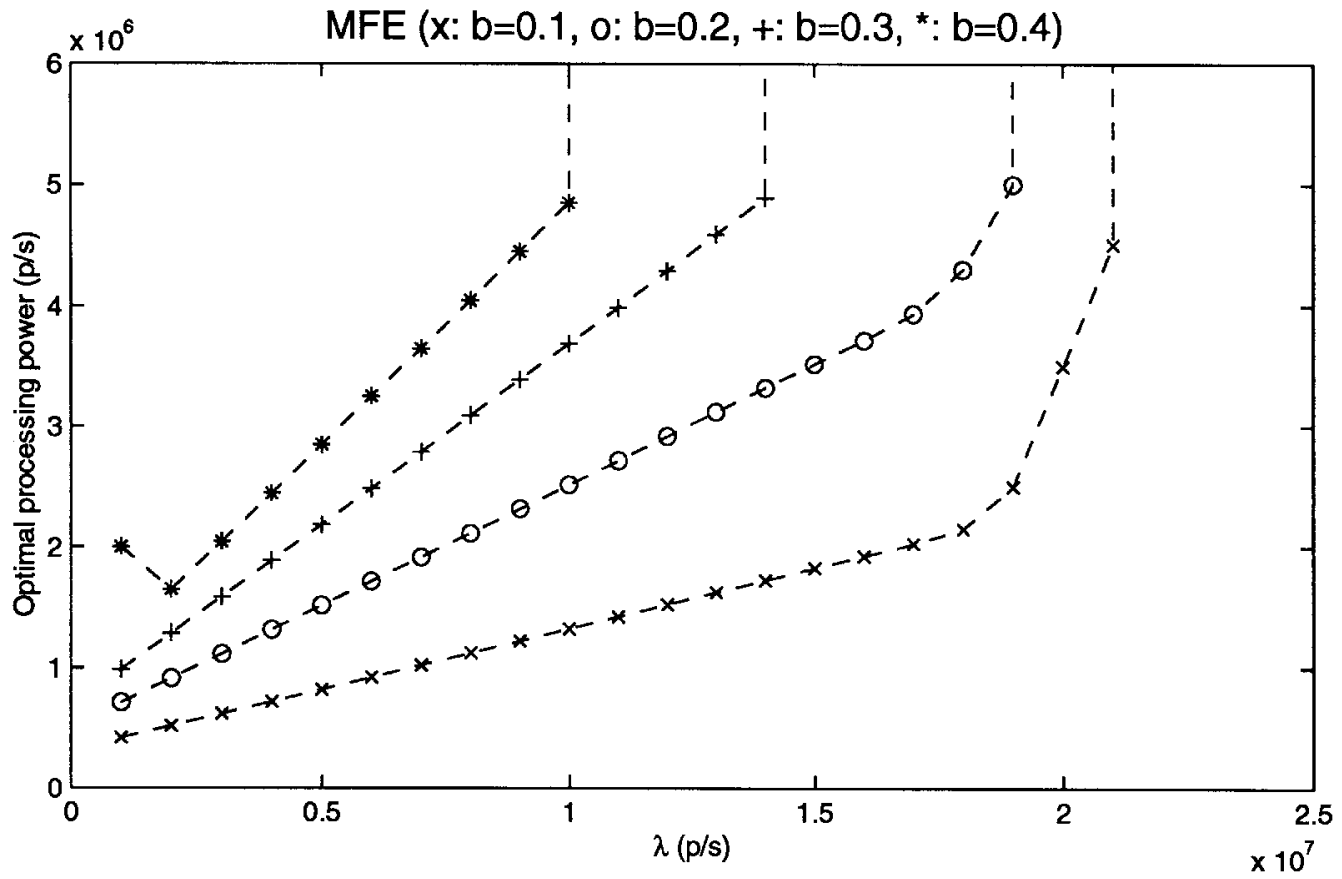

(b)

Fig. 8. Optimal processing power when $b$ is varied $(a=10)$. (a) LFE. (b) MFE.

capacity of the MFE, i.e., $\lambda \leq 5 \times 10^{6}$. This is because when the cost ratio $a \leq 1$, using LFE's would add considerably to the cost of the router. When $\lambda$ is increased beyond the processing capacity of the MFE, the distributed architecture becomes more viable, and the processing power of the LFE's grows above zero. More importantly, the plots for the MFE in Fig. 5 show that once the cost of the MFE increases beyond that of an LFE (that is, $a>1$ ), the performance of the MFE becomes insensitive to additional increases in the cost ratio. For example, there is hardly any difference in the MFE performance for $a=10$ and $a=100$. This provides a very strong argument against centralized router architectures when the packet load is high, because the MFE performance remains poor even with two orders of magnitude improvement in its processing capacity. Thus, at high packet loads, it is more prudent to invest additional cost in the LFE's. Also, note that this result is also consistent with (10) for the ideal case because the first term of (10) is much smaller than the second term when $a$ is large, indicating that $\mu_{1}^{*}$ is less dependent on $a$ when $a$ is large. Inspecting the slopes of the straight lines between $\lambda=5 \times 10^{6}$ and $\lambda=15 \times 10^{6}$ for $a>1$, one finds that they actually follow the gradients $\left(\partial \mu_{1}^{*} / \partial \lambda\right)=b$ and 


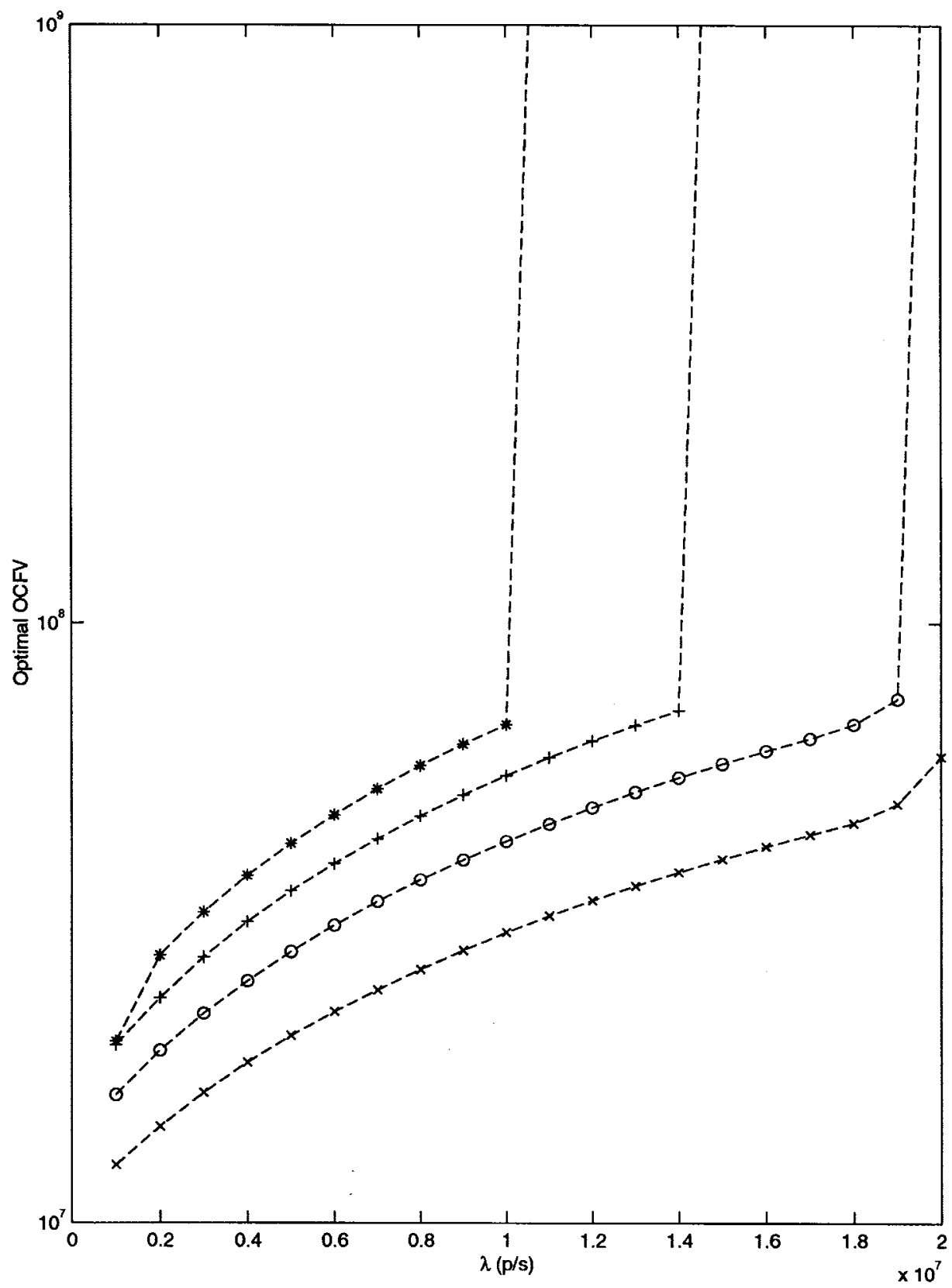

Fig. 9. Optimal OCFV when $b$ is varied $(a=10)$. (x: $b=0.1 ; \circ: b=0.2 ;+: b=0.3 ; *: b=0.4$ ).

$\left(\partial \mu_{2}^{*} / \partial \lambda\right)=(1 / k)$ of the ideal case. Finally, Fig. 6 shows plots for the optimal OCFV defined in (4) for the distributedrouter model. Note that the curve for $a=0.1$ increases more rapidly than the other curves at low $\lambda$, due to the change from a centralized to a distributed architecture. It is also interesting to note that, independent of the cost ratio $a$, all curves approach infinity when $\lambda$ reaches the processing limit of about 20 million $\mathrm{p} / \mathrm{s}$.

2) Impact of the Parameter $b$ : The parameter $b$ was defined earlier as the fraction of route lookups that cannot be resolved locally by an LFE. Fig. 7 shows the optimal $r$ as $b$ takes on four different values $(0.1,0.2,0.3$, and 0.4$)$. The plots show, as expected, that as $b$ increases, the maximum packet rate $\lambda$ that can be handled by the router drops. This is because a larger $b$ means more packets are being forwarded from the LFE's to the central (bottleneck) MFE. As before, the maximum $\lambda$ is determined at the point where $r$ reaches infinity, indicating that there is no feasible solution. The plots still indicate that it is more cost effective to pass route lookups through the LFE's first (i.e., $r=1$ ). The only exception is when $b$ is large $(b=0.4)$ and $\lambda$ is relatively small, in which case it is better to use a centralized architecture (i.e., $r=0$ ). Again, with a large $b$, the LFE's are no longer effective in reducing the processing load of the MFE, and a large fraction of the packets is processed unnecessarily in the LFE's. However, when $\lambda$ is increased beyond the capability of a single MFE, a distributed architecture becomes a necessity. Fig. 8 shows two sets of plots for the optimal processing power of an LFE and an MFE for different values of $b$. In general, the optimal processing power varies linearly with $\lambda$, and the slopes 


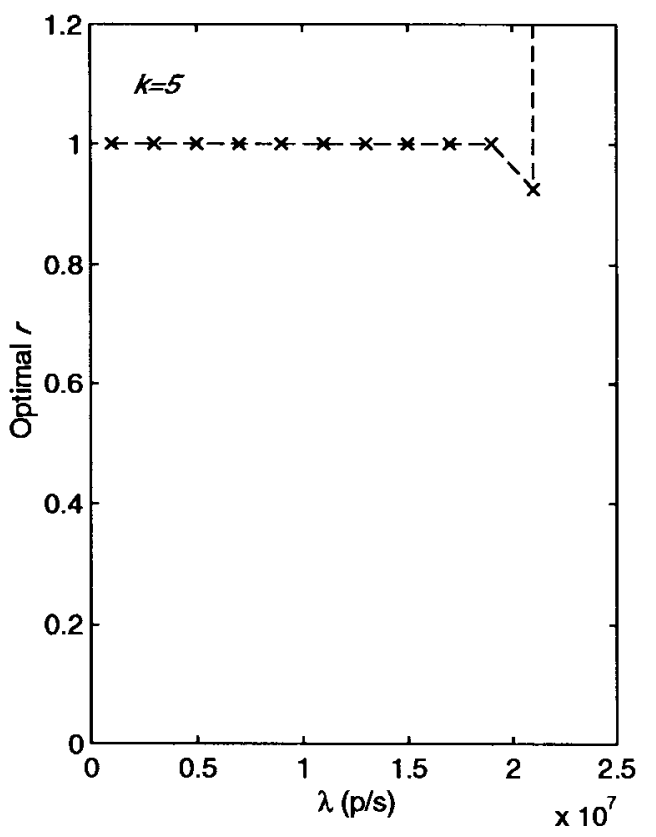

(a)

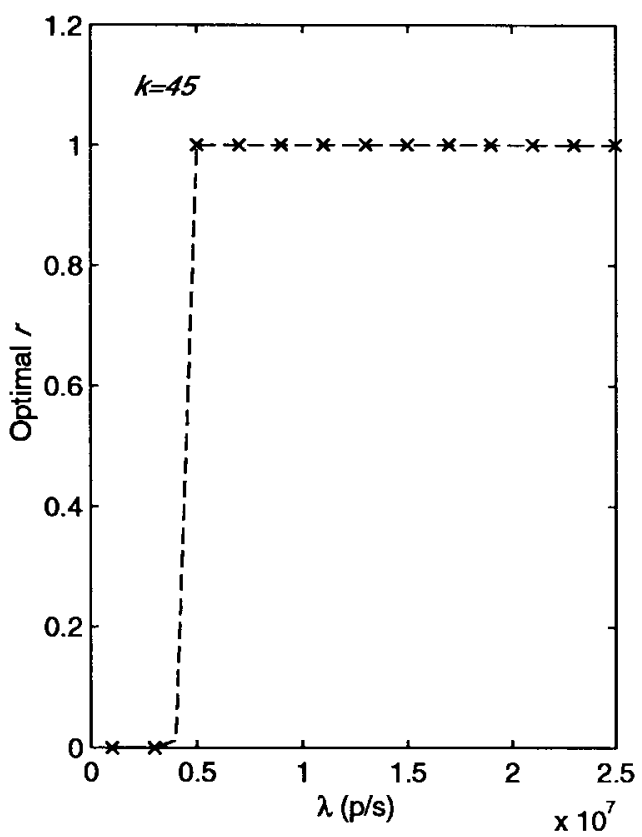

(c)

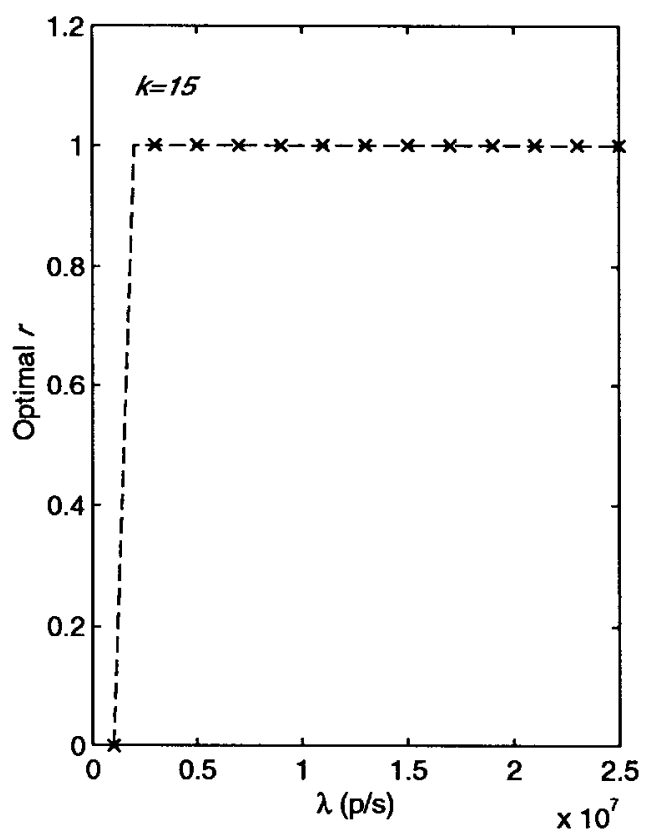

(b)

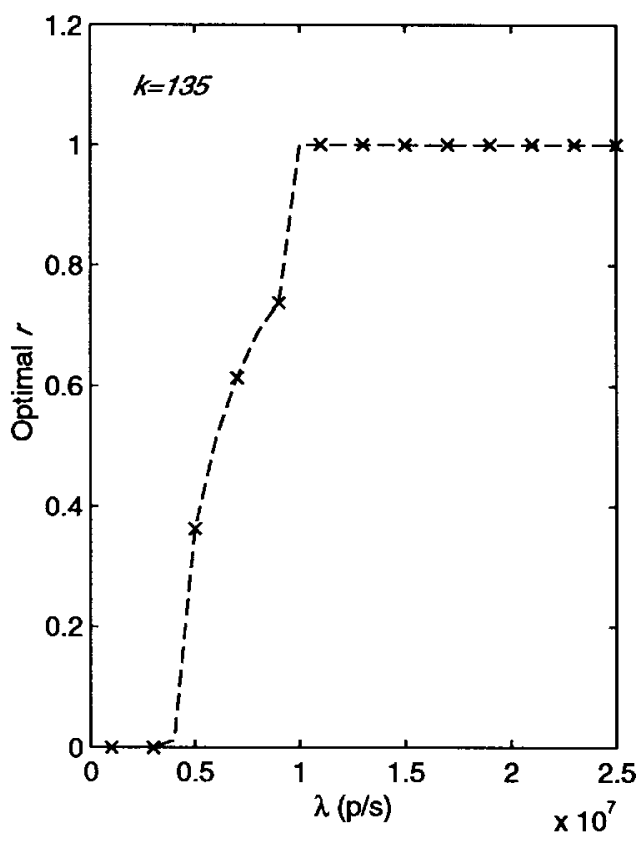

(d)

Fig. 10. Optimal $r$ when $k$ is varied $(a=10)$. (a) $k=5$. (b) $k=15$. (c) $k=45$. (d) $k=135$.

of the plots are aligned with the gradients of (10) and (11) [i.e., $\left(\partial \mu_{1}^{*} / \partial \lambda\right)=b$ and $\left.* \partial \mu_{2}^{*} / \partial \lambda\right)=(1 / k)$ ]. The plots for the LFE in Fig. 8 indicate that the optimal processing power for an LFE is relatively insensitive to changes in $b$, which is also consistent with (11) because the dominant term of the equation is not depend on $b$. However, the processing power of the MFE is strongly affected by $b$ because of the steady growth in packets that must be handled by the MFE when $b$ is not zero. Fig. 9 shows the optimal value for the OCF. As $b$ increases, the optimal value for the OCF goes up to infinity at smaller values of $\lambda$.

3) Impact of the Parameter $k$ : The parameter $k$ denotes the number of LFE's used in the distributed router. Fig. 10 shows the optimal $r$ as $k$ takes on different values $(5,15,45$, and 135). Again, when $\lambda$ is large, it is better to pass the route lookups through the corresponding LFE's first (i.e., $r=1$ ). However, as $k$ gets larger, it becomes more cost effective to use the centralized architecture up to a certain value of $\lambda$. This is because as more LC's are added, the cost of the distributed architecture will increase beyond the cost of the centralized architecture. For example, when $k=135$ and $\lambda$ is in the range of 5-10 million $\mathrm{p} / \mathrm{s}$, a partially centralized architecture is more cost effective (i.e., $0<r<1$ ). In other words, when $k$ is relatively large, the most cost-effective solution mandates the use of LFE's with lower processing power and an MFE with a higher processing power. This observation is substantiated by 


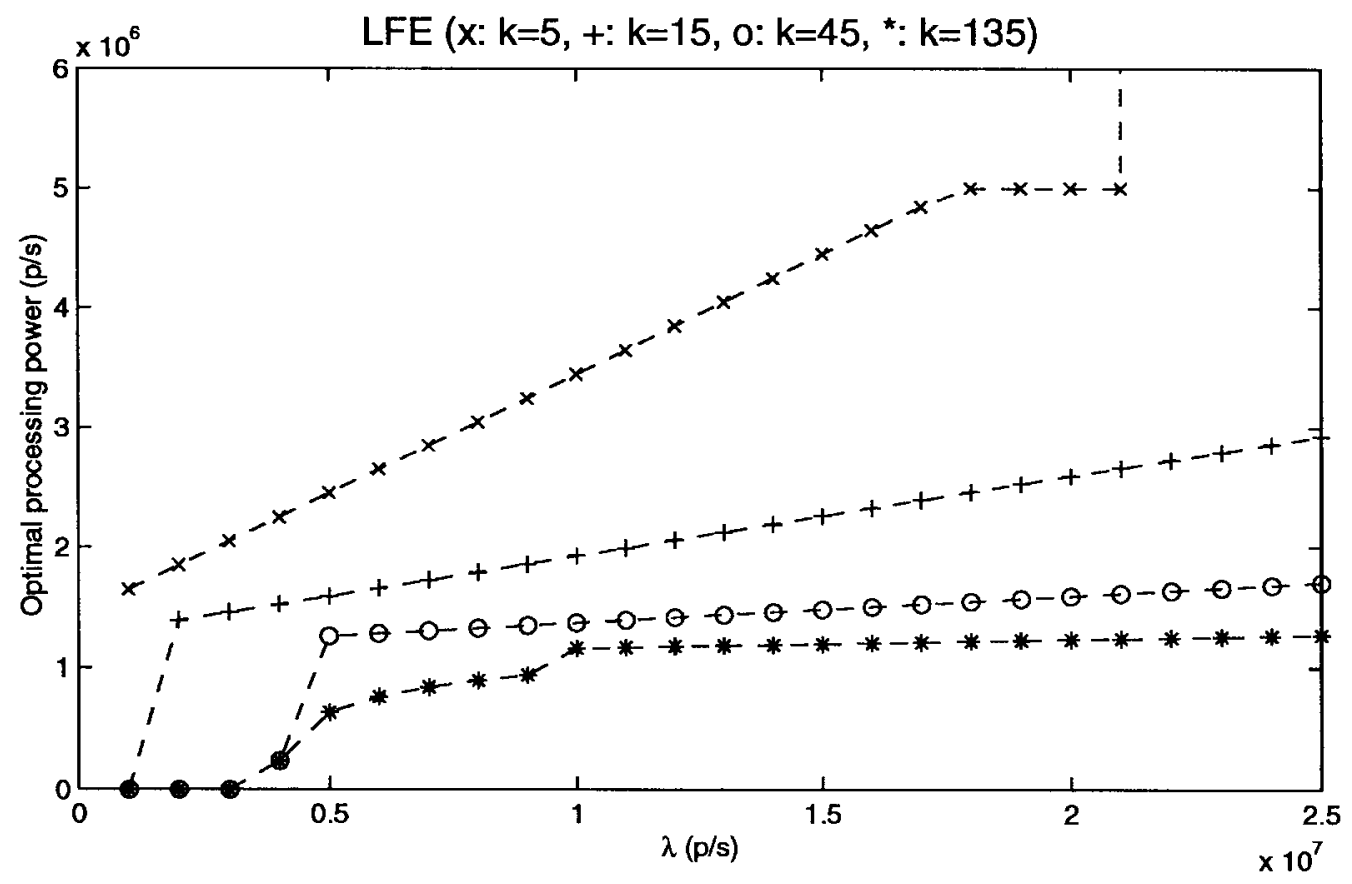

(a)

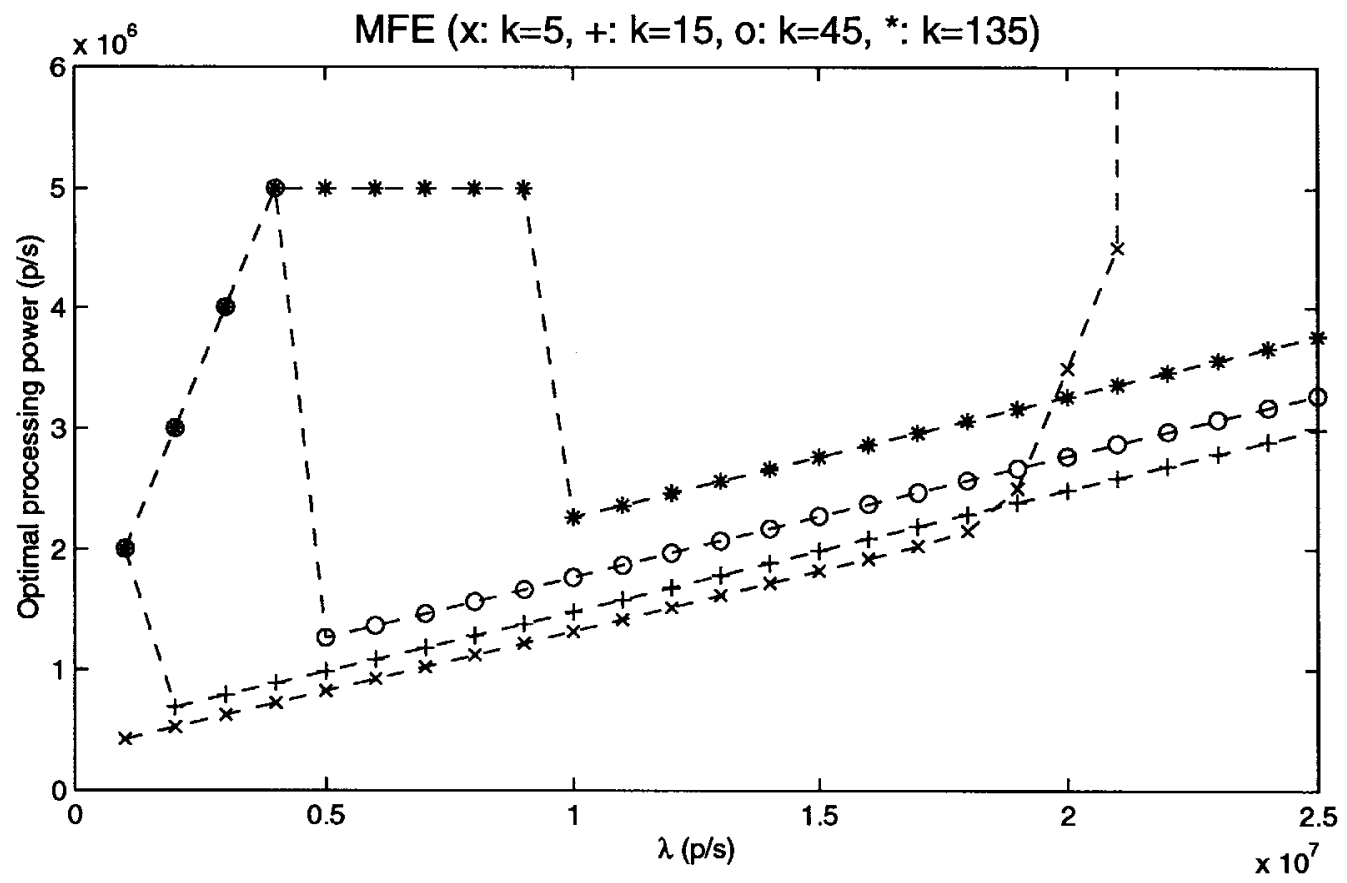

(b)

Fig. 11. Optimal processing power when $k$ is varied $(a=10)$. (a) LFE. (b) MFE.

the plots of Fig. 11, which show the optimal processing power of an LFE and the MFE for different values of $k$. Clearly, the optimal processing power of an LFE decreases as $k$ increases, while the optimal power of the MFE increases with $k$. It is also interesting to observe that when $k$ is large (e.g., $k=135$ ), small increases in the processing power of an LFE leads to drastic drops in the required processing power of the MFE. This can be seen in Fig. 11 near the $\lambda=10 \times 10^{6}$ point for $k=135$, where a small increase in the LFE's power leads to a sharp drop in the required power of the MFE. Similar drastic drops in $\mu_{1}^{*}$ can be observed near $\lambda=2,5$, and 10 million $\mathrm{p} / \mathrm{s}$ for $k=15,45$, and 135 , respectively, due to the change from a centralized or partially centralized architecture to a truly distributed architecture (i.e., $r=1$ ). Although somewhat expected, these results demonstrate the validity of our model in predicting such changes in router performance. Again, Fig. 11 shows that the optimal processing power for an LFE and the MFE varies linearly with $\lambda$ (when $\lambda$ is large), and the slope of the curves are consistent with that gradients of (10) and (11).

Additional interesting observations can be made using Fig. 12, which shows the optimal value for the OCF. First, 


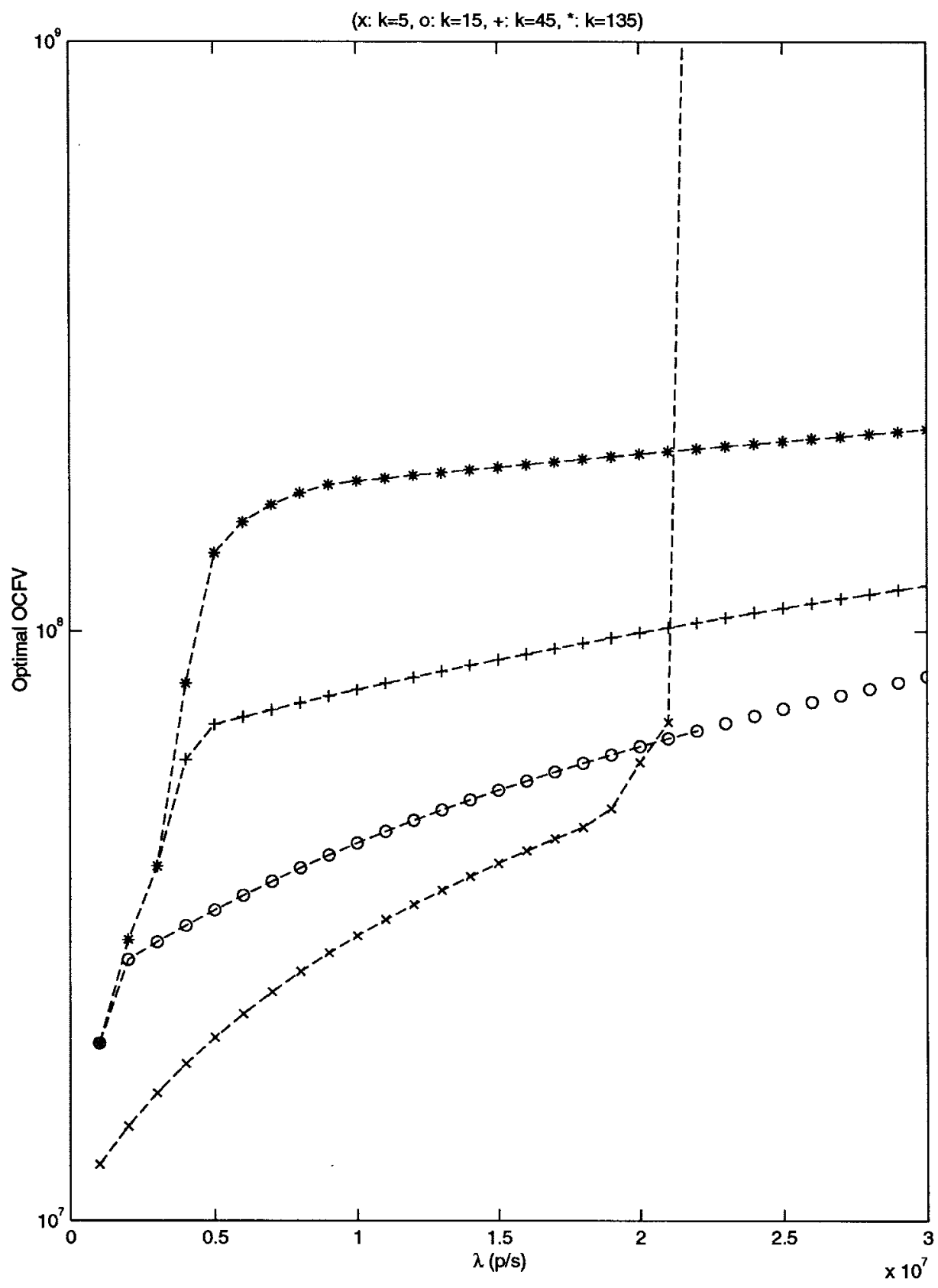

Fig. 12. Optimal OCFV when $k$ is varied $(a=10)$.

note that for $k=5$, the router can only process up to about 20 million $\mathrm{p} / \mathrm{s}$ (or routes), but higher maximum packet rates can be achieved for larger values of $k$. The plots also indicate that the OCFV slope flattens out for larger values of $k$, which means that the optimal OCFV becomes much less sensitive to changes in the packet load $\lambda$. This implies that a large-scale distributed router will be able to increment its packet-processing capacity by several folds without significantly incrementing its cost beyond the initial investment.

\section{B. Parallel-Router Architecture}

The parallel router of Fig. 3 is the second class of router architectures that we consider in this paper. This section provides numerical results based on the concepts presented in Sections II-B and III-B. The parallel-router architecture employs a parallel arrangement of equal-power MFE's and a set of LC's that are capable of performing IP switching on incoming packets (see [5] and Section II-B for details). We start by considering the case when IP switching is not supported in the LC's, i.e., $b=1$. The optimal processing-power curve for a typical MFE is shown in Fig. 13. The saw-tooth pattern is due to the fact that the optimal processing power results either from increasing the power of each individual MFE or by adding a new MFE. When a new MFE is added, the overall processing power of the router is increased, but the effective processing power of the individual MFE's may actually drop because a new MFE handles the additional load. Fig. 14 shows a plot of the optimal number of MFE's required by the parallel architecture as a function of the packet processing rate $\lambda$. The slope of the staircase graph in Fig. 14 indicates that an 


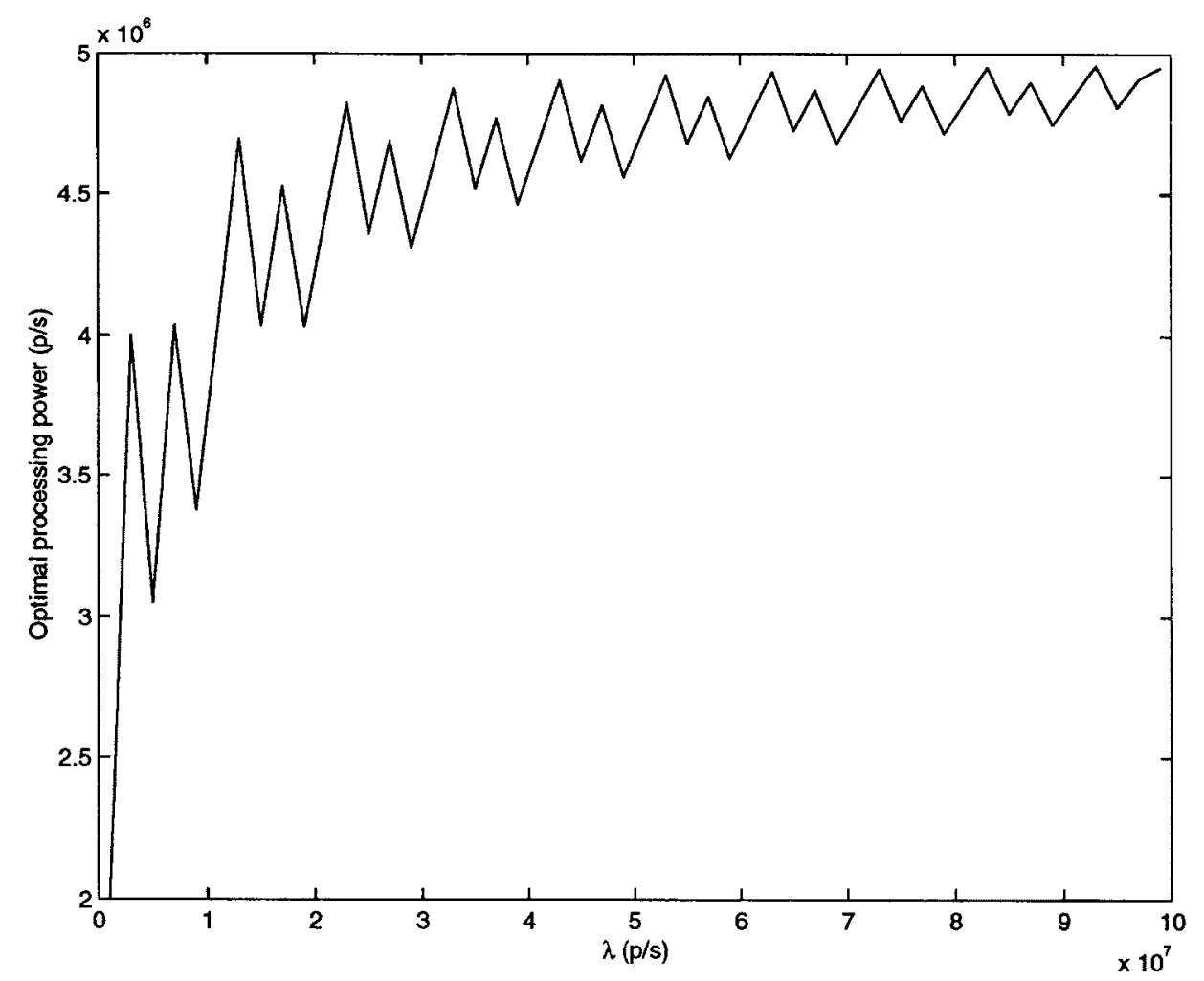

Fig. 13. Optimal processing power of each MFE for the parallel architecture.

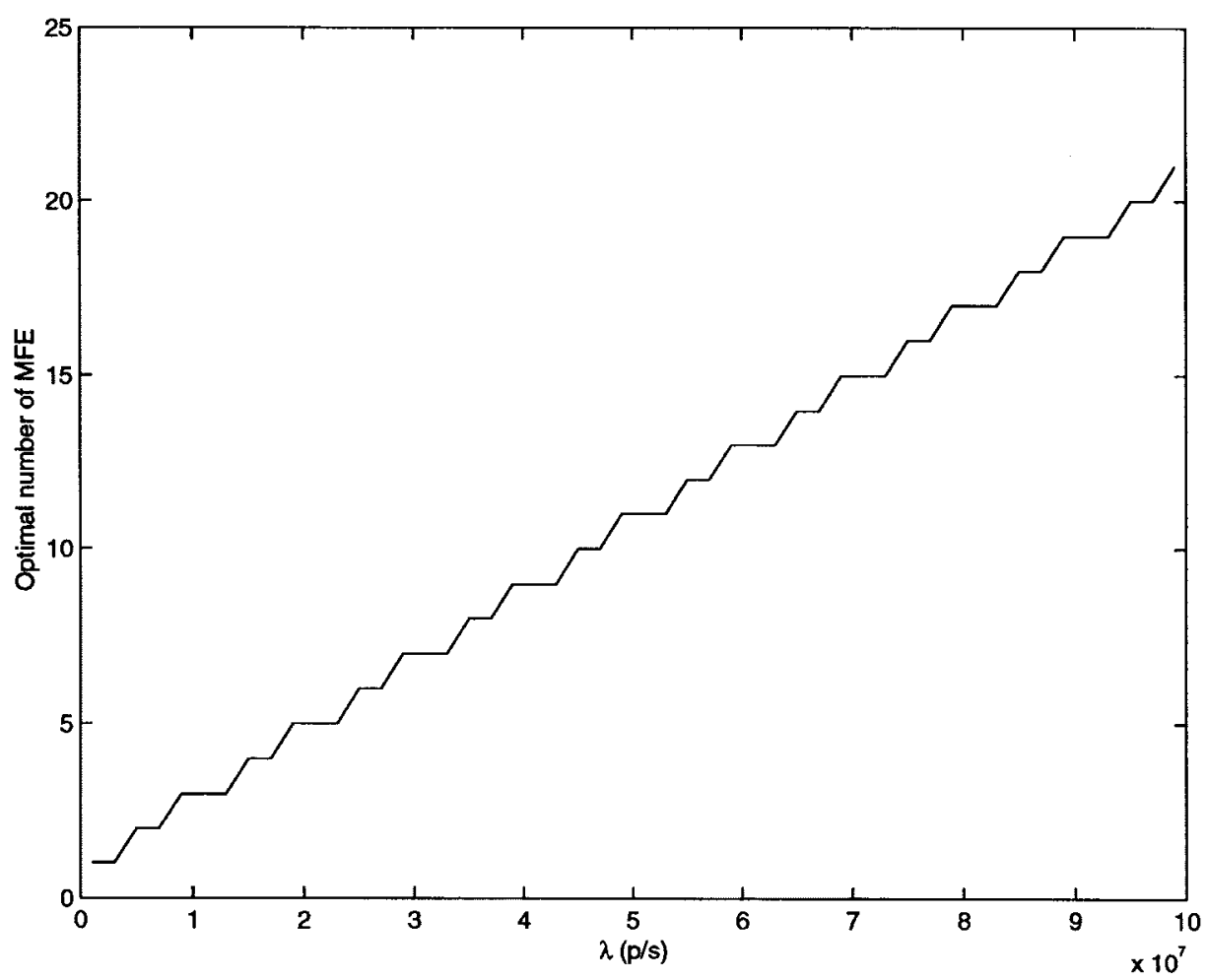

Fig. 14. Optimal number of MFE's for the parallel architecture.

additional MFE is required whenever $\lambda$ increases by about 4 million $\mathrm{p} / \mathrm{s}$. The plot also demonstrates the true scalability of this approach, since processing power can be increased linearly to meet the increased packet-processing load. However, this is only true as far as the MFE's are concerned. In the parallel- router approach, it is more likely that the interconnection fabric or the buffering resources will become the bottlenecks before the MFE's do.

Fig. 15 compares the optimal OCFV of the parallel router with that of the distributed-router architecture. Under our 


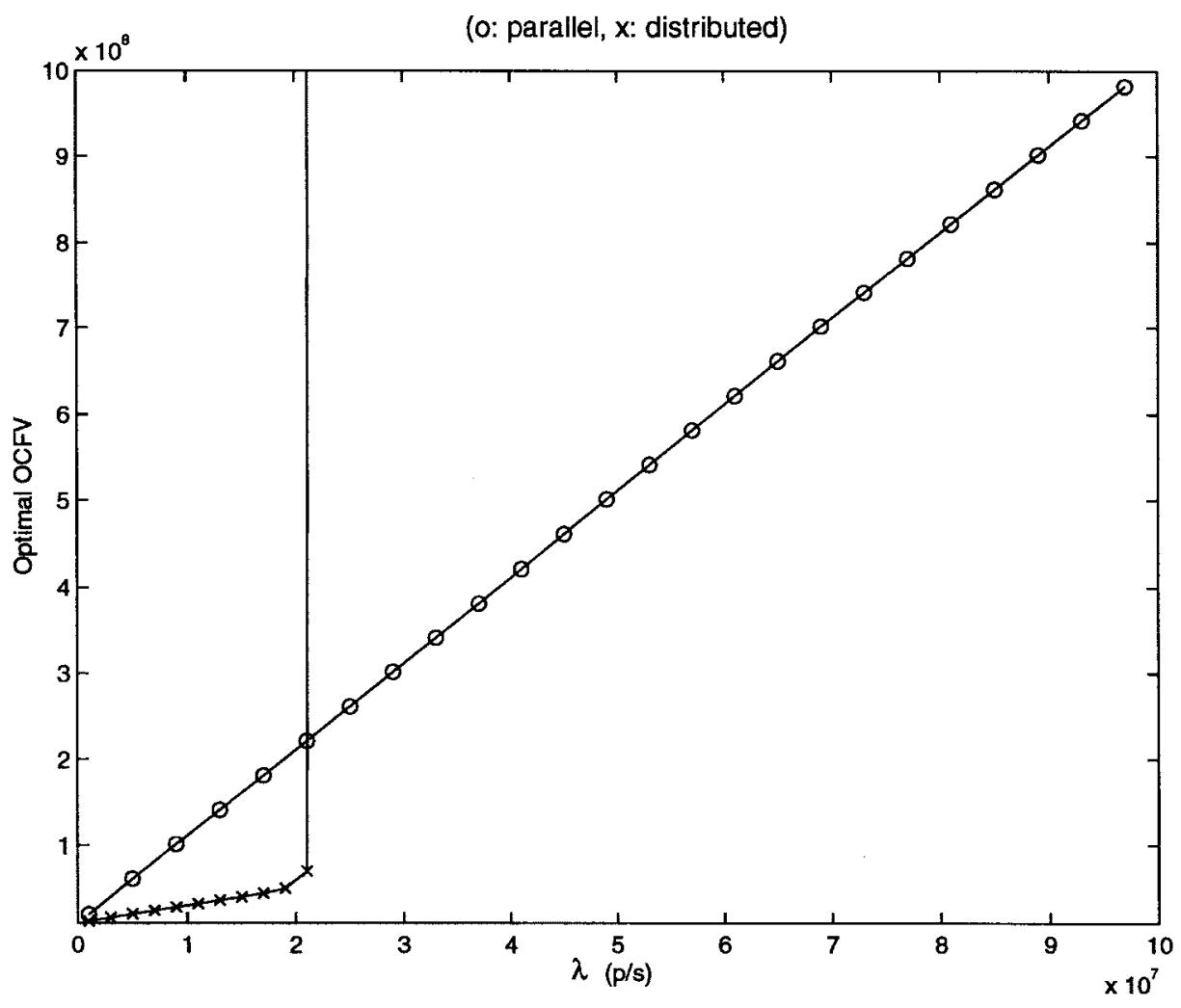

Fig. 15. Comparison of OCFV between distributed and parallel architecture.

assumptions, the cost of the parallel architecture can be much higher than that of the distributed architecture, and the cost difference is larger for larger $\lambda$. This is because the distributed router employs less expensive LFE's to reduce the processing load of the centralized MFE while the parallel router adds more MFE's to increase its packet-processing rate. However, this is true up to the point when the distributedrouter architecture becomes limited by the processing power of the LFE's and the MFE. In our example, this happens at an aggregate packet rate of about 20 million p/s. Beyond that, the scalable parallel-router architecture becomes the only viable solution.

Finally, because the parallel-router architecture provides a scalable solution for very large packet rates, it is worth investigating whether deploying IP switching in the LC's would provide a more cost-effective solution. Indeed, Fig. 15 provides some insight into this problem. Based on the slope of the OCFV curve of the parallel router, one can deduce that the cost of the parallel MFE's is given by $c_{1} \lambda$. To find out, assume that the total cost of deploying IP switching in all LC's is $C_{\mathrm{IPS}}$, and recall that IP switching effectively reduces the packet arrival rate on the MFE's from $\lambda$ to $b \lambda$. Then, a parallel-router architecture with IP switching is more cost effective than one without IP switching if

$$
C_{\operatorname{IPS}}+c_{1} b \lambda<c_{1} \lambda
$$

This inequality can be rearranged to yield

$$
\frac{C_{\mathrm{IPS}}}{c_{1} \lambda}<-b
$$

The previous equation can be used to provide a "figure of merit" for evaluating design alternatives. As an example, consider the case when $b=0.3$, which means that $70 \%$ of the packets can be switched between LC's without going through any MFE [5]. According to (20), IP switching should be deployed if the additional cost $C_{\mathrm{IPS}}$ is less than $0.7 c_{1} \lambda$, i.e., less than $70 \%$ of the total cost of all MFE's. This may indeed be possible in many technologies.

\section{CONCLUSIONS}

This paper presented a new framework for evaluating the cost and performance tradeoffs of distributed- and parallelrouter models. Each of these models is a true representative of commercial routers that have been deployed recently or are still under development. The distributed architecture employs distributed processing, a hardware-based route lookup in the LC's, and a high-speed switching fabric to achieve a very high packet-forwarding rate. The parallel architecture uses a client/server model for handling route lookups, parallel processing, and possibly IP switching in the LC's to achieve scalable high-speed routing. For the distributed-router architecture, numerical results derived from closed-form solutions indicate that it is more cost effective to perform the route lookups in low-cost LFE's colocated with the LC's. This may provide an incentive for the development of hardware-based route lookups in low-cost ASIC's. Assuming the baseline parameters used in Section IV of this paper, the distributed router can forward about 20 millions p/s (or process 20 million routes per second). For the parallel-router architecture, the solution is scalable, and the cost is proportional to the input 
packet rate. The distributed-router architecture has lower cost than the parallel architecture, but it is limited by the processing power of the LFE's and the MFE. However, by incorporating IP switching into the parallel-router architecture, it is possible to further improve its cost effectiveness and packet-forwarding performance.

The key contribution of this paper is the model we have developed for analyzing the cost effectiveness of distributedand parallel-router architectures under certain assumptions. Some of the assumptions were deliberately made not only to simplify analysis, but also to provide useful insight into designing high-speed next-generation routers. Because of the large number of parameters involved, more complicated cost models and packet-arrival models may hide some of the latent complexity issues in large-scale router design. However, our framework can still be applied to more advanced router models. A very useful extension to this work would be to derive more elaborate cost functions that include other technological and/or operational costs. Using more elaborate traffic models may yield more precise insight but we do not expect major surprises there, other than our results may be a bit optimistic because the LC's and the forwarding engines have been uniformly loaded with Poisson traffic.

\section{ACKNOWLEDGMENT}

The authors would like to acknowledge the valuable comments from anonymous referees who have helped to improve the content of this paper.

\section{REFERENCES}

[1] S. Keshav and R. Sharma, "Issues and trends in router design," IEEE Comm. Mag., pp. 144-151, May 1998.

[2] V. P. Kumar, T. V. Lakshman, and D. Stiliadis, "Beyond best effort: Router architectures for the differentiated services of tommorrow's Internet," IEEE Comm. Mag., pp. 152-164, May 1998.

[3] A. Asthana et al., "Toward a gigabit IP router," J. High Speed Networks, vol. 1, no. 4, pp. 281-288, 1992.

[4] C. Partridge, P. P. Carvey, E. Burgess, I. Castineyra, T. Clarke, L. Graham, M. Hathaway, P. Herman, A. King, S. Kohalmi, T. Ma, J. McCallen, T. Mendez, W. C. Milliken, R. Pettyjohn, J. Rokosz, J. Seeger, M. Sollins, S. Storch, B. Tober, G. D. Troxel, D. Waitzman, and S. Winterble, "A 50-Gb/s IP router," IEEE/ACM Trans. Networking, vol. 6, pp. 237-248, June 1998.

[5] P. Newman, G. Minshall, and T. L. Lyon, "IP switching-ATM under IP," IEEE/ACM Trans. Networking, vol. 6, pp. 117-129, Apr. 1998.

[6] H. M. Ahmed, R. Callon, A. G. Malis, and J. Moy, "IP switching for scalable IP services," Proc. IEEE, vol. 85, pp. 1984-1997, Dec. 1997.

[7] Y. Rekhter, B. Davie, E. Rosen, G. Swallow, D. Farinaccl, and D. Katz, "Tag switching architecture overview," Proc. IEEE, vol. 85, pp. 1973-1983, Dec. 1997.

[8] F. Baker, "Requirements for IP version 4 routers," RFC 1812, June 1995.

[9] A. Borodnik et al., "Small forwarding tables for fast route lookups," in Proc. ACM SIGCOMM 97, Cannes, France, pp. 3-14.

[10] P. Gupta, S. Lin, and N. McKeown, "Routing lookups in hardware at memory access speeds," in Proc. IEEE Infocom 98, pp. 1240-1247.

[11] D. C. Feldmeier, "Improving gateway performance with a routing table cache," in Proc. IEEE Infocom'88, New Orleans, LA, pp. 298-307.

[12] A. J. McAuley and P. Francis, "Fast routing table lookup using CAM's," in Proc. IEEE Infocom, San Francisco, CA, vol. 3, Mar. 1993, pp. $1382-1391$.

[13] M. Waldvogel, G. Varghese, J. Turner, and B. Plattner, "Scalable high speed IP route lookups," in Proc. ACM Sigcomm'97, Cannes, France, pp. 25-37.

[14] L. Kleinrock, Queueing Systems, vol. 1. New York: Wiley, 1975.

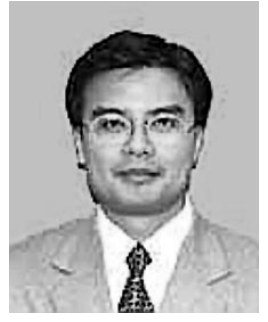

Henry C. B. Chan received the B.A. and M.A. degrees from the University of Cambridge, Cambridge, U.K., and the Ph.D. degree from the University of British Columbia (UBC), Vancouver, B.C., Canada, in 1997.

Between 1988 and 1993, he worked with Hongkong Telecom and was a Visiting Lecturer at Hong Kong Polytechnic University. During his graduate studies, he was a Research and Teaching Assistant in the Communication Research Group, UBC. Between October 1997 and August 1998, he worked with $\mathrm{BC}$ Tel Advanced Communications on the development of high-speed networking technologies and ATM-based services. During the same period, he was also a part-time Postdoctoral Research Fellow, UBC Currently, he is an Assistant Professor in the Department of Computing, Hong Kong Polytechnic University, and he is studying for an M.B.A. degree. His research interests include high-speed networks, Internet technologies, wireless ATM, electronic commerce and personal communications system.

Dr. Chan is a member of ACM and a member of the Chartered Institute of Marketing. He was awarded the NSERC Scholarship from the Government of Canada, the UBC Graduate Fellowship, and the BC TEL Graduate Scholarship. He was the winner of the Institute of Electrical Engineering (Hong Kong Centre) Younger Member Paper Contest in 1992.

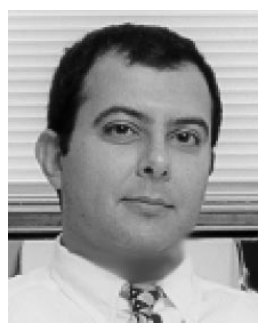

Hussein M. Alnuweiri (S'83-M'83) received the B.Sc. and MA.Sc. degrees from the University of Petroleum and Minerals, Dhahran, Saudi Arabia, in 1983 and 1984, respectively, and the Ph.D. degree in computer engineering from the University of Southern California, Los Angeles, in 1989.

From 1989 to 1991, he was with the Faculty of the Computer Engineering Department at the University of Petroleum and Minerals. Since August 1991, he has been with the Department of Electrical and Computer Engineering, University of British Columbia, Vancouver, B.C., Canada, where he is now an Associate Professor and a member of the Centre for Integrated Computer Systems Research. His main research interests include multimedia communications over packet networks, including IP-based and ATM networks, packet-switching architectures, and embedded system design for video and image processing. He has contributed to over 75 journal and conference papers and has two U.S. patents.

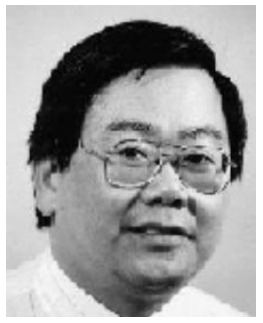

Victor C. M. Leung (S'75-M'79-SM'97) received the B.A.Sc. (with honors) and Ph.D. degrees in electrical engineering from the University of British Columbia (UBC), Vancouver, B.C., Canada, in 1977 and 1981, respectively.

From 1981 to 1987 , he was a Senior Member of Technical Staff, Microtel Pacific Research Ltd., specializing in the planning, design, and analysis of satellite communication systems. He also held a part-time position as Visiting Assistant Professor, Simon Fraser University, in 1986 and 1987. In 1988 , he was a Lecturer in the Department of Electronics, Chinese University of Hong Kong. He joined the Department of Electrical Engineering, UBC, in 1989, where he is now an Associate Professor and a member of the Centre for Integrated Computer Systems Research. He is also a Project Leader in the Canadian Institute for Telecommunications Research. His research interests are in the areas of architectural and protocol design and performance analysis for computer and telecommunication networks, with applications in satellite, mobile, and personal communications and high-speed networks.

Dr. Leung is a voting member of ACM. He was awarded the APEBC Gold Medal as the head of the graduating class at UBC. He was a recipient of the Natural Sciences and Engineering Research Council Postgraduate Scholarship. 